\title{
Statistical theory of catalytic hydrogen oxidation processes. Basic equations
}

\author{
Kostrobij P. P. ${ }^{1}$, Markovych B. M. ${ }^{1}$, Ryzha I. A. ${ }^{1}$, Tokarchuk M. V. ${ }^{2,1}$ \\ ${ }^{1}$ Lviv Polytechnic National University, \\ 12 S. Bandera Str., 79013, Lviv, Ukraine \\ ${ }^{2}$ Institute for Condensed Matter Physics of NAS of Ukraine, \\ 1 Svientsitskii Str., 79011, Lviv, Ukraine
}

(Received 16 October 2020; Revised 3 March 2021; Accepted 17 March 2021)

\begin{abstract}
A statistical description for the processes of catalytic hydrogen oxidation is proposed taking into account the reaction-diffusion processes for magnetoactive ions and atoms adsorbed on the metal surface. The basic non-Markov transfer equations are obtained for the abbreviated description parameters of reaction-diffusion processes for magnetoactive ions and atoms adsorbed on the metal surface in the method of nonequilibrium statistical Zubarev operator. Weakly nonequilibrium reaction-diffusion processes are also considered.
\end{abstract}

Keywords: reaction-diffusion processes, non-Markov equations, the method of nonequilibrium statistical operator.

2010 MSC: $82 \mathrm{C} 05,82 \mathrm{C} 70$

DOI: $10.23939 / \mathrm{mmc} 2021.02 .267$

\section{Introduction}

Environmental problems, which have recently become more acute and are largely associated with the negative impact of carbon monoxide (CO) on the one hand and significant interest in hydrogen energy on the other, pose the task of finding the latest efficient (nano-, sub-atomic) catalysts containing transition metals [1-17] for $\mathrm{CO}$ or $\mathrm{H}$ oxidation. Experimental development of such catalysts requires significant financial costs, but the predictability of their effective operation is quite limited.

The nature of surface phenomena in catalytic reactions is primarily due to the fact that the catalyst surface is magnetoactive (because of the electronic structure of the surface) in such reactions. Inhomogeneous magnetic fields created by magnetic spins of electrons localized on the surface of transition metals (Fe, Ni, Ru, Pt, Pd etc.) affect the processes of surface diffusion of particles (molecules, atoms, ions), which are magnetic dipoles on the surface. Of particular interest are the studies of diffusion and chemisorption processes for induced magnetic dipoles adsorbed on the surface of transition metals [6,18-22], which are effective catalysts. They are relevant because the analysis of semiphenomenological [23-27] and statistical models (the "lattice gas" models) [28] for description of CO oxidation kinetics shows that the surface quantum nature and interaction of $\mathrm{CO}, \mathrm{O}_{2}$ molecules and oxygen atoms with the catalyst surface both have a magneto-dipole nature and can cause the formation of CO clusters on the catalyst surface [29]. Even more interesting are the processes of hydrogen oxidation on the surface of metals, which are actively studied [30-34]. In particular, it is shown in [30] that the process of hydrogen oxidation is characterized by the presence of chemical current caused by the electron transfer $[32,33]$ and formation of bound states on the catalyst surface with electrons and reagents.

In this paper, a quantum-classical model is considered for describing the reaction-diffusion processes for magnetoactive ions and atoms adsorbed on the metal surface. A Hamiltonian of the model is constructed that takes into account the magneto-dipole nature of ions and atoms interaction and chemical catalytic bond with the surface of magnetoactive metal. In order to describe statistically and obtain reaction-diffusion equations, the main parameters of the abbreviated description are determined in the method of nonequilibrium statistical Zubarev operator [35-38]. 


\section{Inhomogeneous transfer equations for description of reaction-diffusion processes for magnetoactive atoms adsorbed on the metal surface}

The processes of dissociation and adsorption of $\mathrm{O}_{2}$ on $\mathrm{Pt}$ are considered as the following successive processes. At $T \leqslant 45 \mathrm{~K}$ there is " $\mathrm{O}_{2}$ " physical adsorption on the surface; in the temperature range of

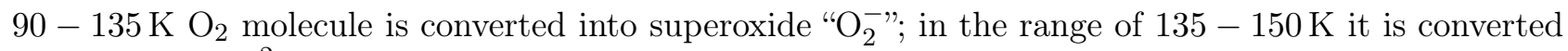
into peroxide " $\mathrm{O}_{2}^{2-}$ ", and then at higher temperatures to oxygen ion vapors " $\mathrm{O}^{-}+\mathrm{O}^{-}$". Schematically, these processes can be presented as follows:

$$
\mathrm{O}_{2}+2 e^{-} \leftrightarrow " \mathrm{O}_{2}+2 e^{-"} \leftrightarrow " \mathrm{O}_{2}^{*}+2 e^{-"} \leftrightarrow " \mathrm{O}_{2}^{2-"} \leftrightarrow " 2 \mathrm{O}^{-"} "
$$

The process of hydrogen molecules adsorption is considered as formation of the bound state hydrogen atom-electron on the surface of platinum:

$$
\mathrm{H}_{2}+2 e^{-} \Leftrightarrow " \mathrm{H}_{2}+2 e^{-”} \Leftrightarrow " 2 \mathrm{H}^{-”} .
$$

Then the oxidation process takes place first with formation of $\mathrm{OH}^{-}$group and release of the electron

$$
" \mathrm{O}^{-}+\mathrm{H}^{-} " \Leftrightarrow\left[" \mathrm{OH}^{-}+e^{-"}\right]^{\neq} \Leftrightarrow \text { " } \mathrm{OH}^{-}+e^{-"}
$$

and the next stage - formation of a water molecule and release of two electrons:

$$
" \mathrm{OH}^{-}+\mathrm{H}^{-} " \Leftrightarrow\left[" \mathrm{OH}^{-}+\mathrm{H}+e^{-"}\right]^{\neq} \Leftrightarrow \text { " } \mathrm{H}_{2} \mathrm{O}^{*}+2 e^{-"},
$$

Here symbol $[\ldots]^{\neq}$denotes the transition state and symbol "** denotes the adsorbed state.

Anions of $\mathrm{O}^{-}, \mathrm{H}^{-}, \mathrm{OH}^{-}$in adsorbed state on the platinum surface are magnetic dipoles in reactions (1)-(4) and $\mathrm{H}_{2} \mathrm{O}^{*}$ is the dipole in adsorbed state. Therefore, from the point of view of model description, there is a system of magnetic dipole particles that fill the volume above the metal surface and can be adsorbed on it, interacting with the electron subsystem of metal surface.

For generality, we consider a system with $\bar{n}$ different sorts of magnetoactive particles (molecules, atoms, anions, electrons) with $N_{a}$ number particles of sort $a(a=1, \ldots, \bar{n})$. The Hamiltonian of the system is given as the sum of classical $H_{L}$ and quantum $\hat{H}_{q}(t)$ parts:

$$
\hat{H}(t)=H_{L}+\hat{H}_{q}(t)
$$

where

$$
H_{L}=\sum_{a} \sum_{j=1}^{N_{a}} \frac{p_{j}^{2}}{2 m_{a}}+\frac{1}{2} \sum_{a b} \sum_{j \neq l} \Phi_{a b}\left(r_{j l}\right)+V_{a d}
$$

is the classical part of Hamiltonian, which describes the system of magnetoactive particles as a simple classical gas, the atoms of sort $a$ of which can be adsorbed on the magnetoactive surface of metal with an adsorption potential $V_{a d} . r_{j l}$ is the distance between $j$ and $l$ particles, $\boldsymbol{p}_{j}$ and $m_{a}$ are the vector momentum and the mass of magnetoactive particle of sort $a, \Phi_{a b}\left(r_{j l}\right)$ is the pair potential of classical interaction between magnetoactive particles of sort $a$ and $b$, which can be modeled by the LennardJones potential. Values $a, b, \ldots$ in the sums are the indexes of the sort. An example of adsorption potential $V_{a d}$ in the right part of the Hamiltonian is the Morse potential

$$
V_{a d}(z)=D_{e}\left[1-e^{C\left(z-z_{e}\right)}\right]^{2},
$$

where $z$ is the coordinate in direction perpendicular to the surface, $D_{e}$ is the depth of potential well for the equilibrium value of $z_{e}$ coordinate, $C$ is a constant. The quantum part of the Hamiltonian $\hat{H}_{q}(t)$

$$
\hat{H}_{q}(t)=\hat{H}_{e}(t)+\hat{H}_{S}(t)
$$


contains the contribution of electron subsystem:

$$
\hat{H}_{e}(t)=\sum_{j=1}^{N_{e}} \frac{\left(\boldsymbol{p}_{j}-\frac{e}{c} \boldsymbol{A}(\boldsymbol{r} ; t)\right)^{2}}{2 m_{e}}+V_{e e},
$$

with potential of electron-electron interaction $V_{e e}$ ( $e$ is the electron charge, $c$ is the speed of light, $\boldsymbol{A}(\boldsymbol{r} ; t)$ is the vector potential of electromagnetic field generated by electrons and magnetoactive particles in the system) and contribution $\hat{H}_{S}(t)$ describing a system of magnetoactive particles with spins $\boldsymbol{S}_{j}$ in the inhomogeneous magnetic field $\boldsymbol{B}^{\prime}(\boldsymbol{r} ; t)$ and in the field created by $N_{m}$ magnetoactive particles of the surface with coordinates $\boldsymbol{R}_{f}$ and spins, that can be presented as follows:

$$
\hat{H}_{S}(t)=\hat{H}_{S}-\sum_{a} \int d \boldsymbol{r} \hat{\boldsymbol{M}}_{a}(\boldsymbol{r}) \boldsymbol{B}_{a}(\boldsymbol{r} ; t)
$$

Here $\boldsymbol{B}_{a}\left(\boldsymbol{r}_{j} ; t\right)=\boldsymbol{B}^{\prime}\left(\boldsymbol{r}_{j} ; t\right)+\sum_{f=1}^{N_{m}} J_{a}\left(\boldsymbol{r}_{j}, \boldsymbol{R}_{f}\right) \boldsymbol{\sigma}_{f}$ is the total magnetic field acting on the $j$-th magnetic particle of sort $a ; J_{a}\left(\boldsymbol{r}_{j}, \boldsymbol{R}_{f}\right)$ is the exchange integral for interaction of magnetoactive particles with magnetoactive sites of the metal surface, and

$$
\begin{aligned}
\hat{H}_{S} & =-\frac{1}{2} \sum_{a b} \sum_{j \neq l} J_{a b}^{s h}\left(r_{j l}\right) \boldsymbol{S}_{j} \boldsymbol{S}_{l}-\frac{1}{2} \sum_{a b} \sum_{\alpha \gamma} \sum_{j \neq l} J_{a b}^{l, \alpha \gamma}\left(r_{j l}\right) S_{j}^{\alpha} S_{l}^{\gamma} \\
& =-\sum_{a b} \frac{1}{2} \int d \boldsymbol{r} \int d \boldsymbol{r}^{\prime} J_{a b}^{s h}\left(\boldsymbol{r}, \boldsymbol{r}^{\prime}\right) \boldsymbol{S}_{a}(\boldsymbol{r}) \boldsymbol{S}_{b}\left(\boldsymbol{r}^{\prime}\right)-\frac{1}{2} \sum_{a b} \sum_{\alpha \gamma} \int d \boldsymbol{r} \int d \boldsymbol{r} J_{a b}^{l, \alpha \gamma}\left(\boldsymbol{r}, \boldsymbol{r}^{\prime}\right) S_{a}^{\alpha}(\boldsymbol{r}) S_{b}^{\gamma}\left(\boldsymbol{r}^{\prime}\right)
\end{aligned}
$$

is the quantum part of the Hamiltonian, which describes the system of magnetoactive particles; $\boldsymbol{S}_{a}(\boldsymbol{r})=\sum_{j=1}^{N_{a}} \boldsymbol{S}_{j} \delta\left(\boldsymbol{r}-\boldsymbol{r}_{j}\right)$ is the spin $\boldsymbol{S}_{j}$ density operator of magnetic particles of sort $a$; $\hat{\boldsymbol{M}}_{a}(\boldsymbol{r})=\mu_{a} \boldsymbol{S}_{a}(\boldsymbol{r})$ is the operator of magnetic moment density of magnetoactive particles of sort $a$. $J_{a b}\left(r_{j l}\right)$ is the exchange integral for interaction of magnetoactive particles, which has two components: $J_{a b}^{s h}\left(r_{j l}\right)$ is the short-term magnetic interaction, which can lead to a chemical bond between adsorbed particles of sort $a$ and $b, J_{a b}^{l, \alpha \gamma}\left(r_{j l}\right)$ are the components of tensor for long-range dipole-dipole interaction of adsorbed particles of sort $a, b[22]$ :

$$
J_{a b}^{l, \alpha \gamma}\left(r_{j l}\right)=-\frac{1}{2}\left(g_{L} \mu_{B}\right)^{2}\left(\frac{\delta_{\alpha \gamma}}{\left|\boldsymbol{r}_{j l}\right|^{3}}-\frac{3\left(\boldsymbol{r}_{j}-\boldsymbol{r}_{l}\right)^{\alpha}\left(\boldsymbol{r}_{j}-\boldsymbol{r}_{l}\right)^{\gamma}}{\left|\boldsymbol{r}_{j l}\right|^{5}}\right) .
$$

Here $g_{L}$ is the Lang factor, $\mu_{B}$ is the Bohr magneton, $\alpha, \gamma=\{x, y, z\}$-components. Combining the exchange (Heisenberg) and dipole components, we introduce: $J_{a b}^{\alpha \beta}(\boldsymbol{r})=J_{a b}^{s h}(r) \delta_{\alpha \gamma}+J_{a b}^{l, \alpha \gamma}(\boldsymbol{r})$ with $J_{a b}^{\alpha \gamma}(\boldsymbol{r})$ presented as the sum of diagonal and non-diagonal components:

$$
J_{a b}^{\alpha \gamma}(\boldsymbol{r})=J_{a b}^{1}(r) \delta_{\alpha \gamma}+J_{a b}^{2}(r) r^{\alpha} r^{\gamma},
$$

where

$$
J_{a b}^{1}(r)=J_{a b}^{s h}(r)-\frac{1}{2} \frac{g_{a} g_{b}\left(\mu_{B}\right)^{2}}{r^{3}}, \quad J_{a b}^{2}(r)=\frac{3}{2} \frac{g_{a} g_{b}\left(\mu_{B}\right)^{2}}{r^{5}}
$$

and $g_{a}, g_{b}$ are the Lang factors of particles of sort $a$ and $b$, respectively.

Hamiltonian $\hat{H}_{S}$ can now be written as

$$
\begin{aligned}
\hat{H}_{S} & =-\frac{1}{2} \sum_{a b} \sum_{j \neq l} J_{a b}\left(r_{j l}\right) \boldsymbol{S}_{j} \boldsymbol{S}_{l} \\
& =-\frac{1}{2} \sum_{a b} \sum_{j \neq l} J_{a b}^{1}\left(r_{j l}\right)\left(\boldsymbol{S}_{j} \cdot \boldsymbol{S}_{l}\right)-\frac{1}{2} \sum_{a b} \sum_{j \neq l} J_{a b}^{2}\left(r_{j l}\right)\left(\boldsymbol{r}_{j l} \cdot \boldsymbol{S}_{j}\right)\left(\boldsymbol{r}_{j l} \cdot \boldsymbol{S}_{l}\right) .
\end{aligned}
$$

Mathematical Modeling and Computing, Vol. 8, No. 2, pp. 267-281 (2021) 
In order to describe the diffusional and magnetostrictive processes on the metal surface, we choose the average values of particle number density (molecules, atoms, anions, electrons) $\left\langle\hat{n}_{a}(\boldsymbol{r})\right\rangle^{t}$ and magnetization $\left\langle\boldsymbol{M}_{a}(\boldsymbol{r})\right\rangle^{t}$ as parameters (observed values) of the abbreviated description [39-41]. To study the cluster coverage and correlations, we also include the non-equilibrium structural functions "density - density" $\left\langle\hat{g}_{a b}\left(\boldsymbol{r}, \boldsymbol{r}^{\prime}\right)\right\rangle^{t}$ and "spin-spin" $\left\langle\stackrel{\leftrightarrow}{G} a b\left(\boldsymbol{r}, \boldsymbol{r}^{\prime}\right)\right\rangle^{t}$ in the set of observed values, where $\langle\ldots\rangle^{t}=\operatorname{Sp}(\ldots \rho(t)), \rho(t)$ is the nonequilibrium statistical operator of a system. All dynamic variables and operators corresponding to the observed variables are defined as follows:

$$
\hat{n}_{a}(\boldsymbol{r})=\sum_{j=1}^{N_{a}} \delta\left(\boldsymbol{r}-\boldsymbol{r}_{j}\right)
$$

is the microscopic density of number of magnetoactive particles of sort $a$,

$$
\begin{aligned}
\hat{g}_{a b}\left(\boldsymbol{r}, \boldsymbol{r}^{\prime}\right) & =\hat{n}_{a}(\boldsymbol{r}) \hat{n}_{b}\left(\boldsymbol{r}^{\prime}\right), \\
\hat{G}_{a b}^{\alpha \gamma}\left(\boldsymbol{r}, \boldsymbol{r}^{\prime}\right) & =S_{a}^{\alpha}(\boldsymbol{r}) S_{b}^{\gamma}\left(\boldsymbol{r}^{\prime}\right) .
\end{aligned}
$$

In order to obtain the transfer equations for parameters of the abbreviated description $\left\langle\hat{n}_{a}(\boldsymbol{r})\right\rangle^{t}$, $\left\langle\boldsymbol{M}_{a}(\boldsymbol{r})\right\rangle^{t},\left\langle\hat{g}_{a b}\left(\boldsymbol{r}, \boldsymbol{r}^{\prime}\right)\right\rangle^{t},\left\langle\hat{\leftrightarrow}_{G b}\left(\boldsymbol{r}, \boldsymbol{r}^{\prime}\right)\right\rangle^{t}$, it is necessary to construct the corresponding nonequilibrium statistical operator $\rho(t)$, which satisfies the Liouville equation [35]. To obtain $\rho(t)$, we use the method of nonequilibrium statistical Zubarev operator [35,36], taking into account the design procedure. In the general case, the nonequilibrium statistical operator can be represented as:

$$
\rho(t)=\rho_{q}(t)-\int_{-\infty}^{t} e^{\varepsilon\left(t^{\prime}-t\right)} \hat{T}\left(t, t^{\prime}\right)\left(1-P_{q}\left(t^{\prime}\right)\right) i \hat{L}\left(t^{\prime}\right) \rho_{q}\left(t^{\prime}\right) d t^{\prime}
$$

where

$$
\begin{aligned}
\rho_{q}(t)= & \exp \left\{-\Phi(t)-\beta\left(\hat{H}(t)-\sum_{a} \int \mu_{a}(\boldsymbol{r}, t) \hat{n}_{a}(\boldsymbol{r}) d \boldsymbol{r}\right.\right. \\
& -\sum_{a b} \int d \boldsymbol{r} \int d \boldsymbol{r}^{\prime} \stackrel{\leftrightarrow}{\mu}_{b a}\left(\boldsymbol{r}, \boldsymbol{r}^{\prime} t\right) \bullet \stackrel{\leftrightarrow}{G}_{a b}\left(\boldsymbol{r}, \boldsymbol{r}^{\prime}\right) \\
& \left.\left.-\sum_{a} \int d \boldsymbol{r} \hat{\boldsymbol{M}}_{a}(\boldsymbol{r}) \cdot \boldsymbol{b}_{a}(\boldsymbol{r} ; t)-\sum_{a b} \int d \boldsymbol{r} \int d \boldsymbol{r}^{\prime} \chi_{b a}\left(\boldsymbol{r}, \boldsymbol{r}^{\prime} t\right) \hat{g}_{a b}\left(\boldsymbol{r}, \boldsymbol{r}^{\prime}\right)-\mu_{m} N_{m}\right)\right\}
\end{aligned}
$$

is the relevant statistical operator constructed from the extremum of information entropy of the system at fixed parameters of the abbreviated description $\left\langle\hat{n}_{a}(\boldsymbol{r})\right\rangle^{t},\left\langle\hat{\boldsymbol{M}}_{a}(\boldsymbol{r})\right\rangle^{t},\langle\hat{H}(t)\rangle^{t},\left\langle\stackrel{\leftrightarrow}{G}_{a b}\left(\boldsymbol{r}, \boldsymbol{r}^{\prime}\right)\right\rangle^{t}$, $\left\langle\hat{g}_{a b}\left(\boldsymbol{r}, \boldsymbol{r}^{\prime}\right)\right\rangle^{t}$ and preserving a condition $\operatorname{Sp} \rho_{q}(t)=1$, with $\langle\ldots\rangle^{t}=\operatorname{Sp}[\ldots \rho(t)]$, $\bullet$ denoting the tensor product. Here $\mu_{a}(\boldsymbol{r}, t)$ is the local chemical potential of magnetoactive particles of sort $a$, in the case of electrons $a=e, \mu_{e}(\boldsymbol{r}, t)=\bar{\mu}_{e}(\boldsymbol{r}, t)-e \varphi(\boldsymbol{r}, t)$ is the electrochemical potential, $\bar{\mu}_{e}(\boldsymbol{r}, t)$ is the local chemical potential of electrons, $\varphi(\boldsymbol{r}, t)$ is the scalar potential of electromagnetic field in the system. $\mu_{m}$ is the chemical potential of magnetoactive sites of the metal surface, $\beta=1 / k_{\mathrm{B}} T$ is the inverse temperature, $k_{\mathrm{B}}$ is the Boltzmann constant, $\boldsymbol{b}_{a}(\boldsymbol{r} ; t)$ is the internal magnetic field created by magnetoactive particles of sort $a, \overleftrightarrow{\mu}_{b a}\left(\boldsymbol{r}, \boldsymbol{r}^{\prime} t\right)$ are the Lagrange parameters and have a tensor dimension and are related to the correlation functions "spin density - spin density" $\left\langle\stackrel{\leftrightarrow}{G} a b\left(\boldsymbol{r}, \boldsymbol{r}^{\prime}\right)\right\rangle^{t}, \chi_{b a}\left(\boldsymbol{r}, \boldsymbol{r}^{\prime} t\right)$ are the Lagrange parameters and play the role of chemical potential for complexes of $a b$ reagents and are conjugate to correlation functions "density - density" $\left\langle\hat{g}_{a b}\left(\boldsymbol{r}, \boldsymbol{r}^{\prime}\right)\right\rangle^{t}$.

$$
\begin{aligned}
\Phi(t)= & \ln \operatorname{Sp} \exp \left\{-\beta(t)\left(\hat{H}(t)-\sum_{a} \int \mu_{a}(\boldsymbol{r}, t) \hat{n}_{a}(\boldsymbol{r}) d \boldsymbol{r}-\sum_{a b} \int d \boldsymbol{r} \int d \boldsymbol{r}^{\prime} \stackrel{\leftrightarrow}{\mu}_{b a}\left(\boldsymbol{r}, \boldsymbol{r}^{\prime} t\right) \bullet \stackrel{\leftrightarrow}{G}_{a b}\left(\boldsymbol{r}, \boldsymbol{r}^{\prime}\right)\right.\right. \\
& \left.\left.-\sum_{a} \int d \boldsymbol{r} \hat{\boldsymbol{M}}_{a}(\boldsymbol{r}) \cdot \boldsymbol{b}_{a}(\boldsymbol{r} ; t)-\sum_{a b} \int d \boldsymbol{r} \int d \boldsymbol{r}^{\prime} \chi_{b a}\left(\boldsymbol{r}, \boldsymbol{r}^{\prime} t\right) \hat{g}_{a b}\left(\boldsymbol{r}, \boldsymbol{r}^{\prime}\right)-\mu_{m} N_{m}\right)\right\}
\end{aligned}
$$


is the Masier-Planck functional. $i \hat{L}\left(t^{\prime}\right)$ is the Liouville operator corresponding to Hamiltonian (5) of the system, $P_{q}\left(t^{\prime}\right)$ is the Kawasaki-Ganton projection operator [36], the structure of which depends on the statistical operator (8), $\hat{T}\left(t, t^{\prime}\right)=\exp _{+}\left\{-\int_{t^{\prime}}^{t}\left(1-P_{q}\left(t^{\prime \prime}\right)\right) i \hat{L}\left(t^{\prime}\right) d t^{\prime \prime}\right\}$ is the evolution operator based on the design. The Lagrange multipliers - nonequilibrium thermodynamic parameters $\mu_{a}(\boldsymbol{r}, t)$, $\boldsymbol{b}_{a}(\boldsymbol{r} ; t), \overleftrightarrow{\mu}_{b a}\left(\boldsymbol{r}, \boldsymbol{r}^{\prime} t\right), \chi_{b a}\left(\boldsymbol{r}, \boldsymbol{r}^{\prime} t\right)$ are determined from the corresponding self-agreement conditions:

$$
\begin{gathered}
\left\langle\hat{n}_{a}(\boldsymbol{r})\right\rangle^{t}=\left\langle\hat{n}_{a}(\boldsymbol{r})\right\rangle_{q}^{t}, \quad\left\langle\hat{\boldsymbol{M}}_{a}(\boldsymbol{r})\right\rangle^{t}=\left\langle\hat{\boldsymbol{M}}_{a}(\boldsymbol{r})\right\rangle_{q}^{t}, \\
\left\langle\hat{\leftrightarrow}_{a b}\left(\boldsymbol{r}, \boldsymbol{r}^{\prime}\right)\right\rangle^{t}=\left\langle\stackrel{\leftrightarrow}{G}_{a b}\left(\boldsymbol{r}, \boldsymbol{r}^{\prime}\right)\right\rangle_{q}^{t}, \quad\left\langle\hat{g}_{a b}\left(\boldsymbol{r}, \boldsymbol{r}^{\prime}\right)\right\rangle^{t}=\left\langle\hat{g}_{a b}\left(\boldsymbol{r}, \boldsymbol{r}^{\prime}\right)\right\rangle_{q}^{t} .
\end{gathered}
$$

We substitute the relevant statistical operator into (7) and, performing the action of the Liouville operator on $\rho_{q}(t)$, we obtain for the nonequilibrium statistical operator:

$$
\begin{aligned}
\rho(t)= & \rho_{q}(t)-\sum_{a} \int d \boldsymbol{r}^{\prime} \int_{-\infty}^{t} e^{\varepsilon\left(t^{\prime}-t\right)} \hat{T}\left(t, t^{\prime}\right) \int_{0}^{1} d \tau \rho_{q}^{\tau}\left(t^{\prime}\right) I_{n}^{a}\left(\boldsymbol{r}^{\prime} ; t^{\prime}\right) \rho_{q}^{1-\tau}\left(t^{\prime}\right) \beta \mu_{a}\left(\boldsymbol{r}^{\prime}, t^{\prime}\right) d t^{\prime} \\
& -\sum_{a} \int d \boldsymbol{r}^{\prime} \int_{-\infty}^{t} e^{\varepsilon\left(t^{\prime}-t\right)} \hat{T}\left(t, t^{\prime}\right) \int_{0}^{1} d \tau \rho_{q}^{\tau}\left(t^{\prime}\right) I_{M}^{a}\left(\boldsymbol{r}^{\prime} ; t^{\prime}\right) \rho_{q}^{1-\tau}\left(t^{\prime}\right) \beta \boldsymbol{b}_{a}\left(\boldsymbol{r}^{\prime} ; t^{\prime}\right) d t^{\prime} \\
& -\sum_{a b} \int d \boldsymbol{r} \int d \boldsymbol{r}^{\prime} \int_{-\infty}^{t} e^{\varepsilon\left(t^{\prime}-t\right)} \hat{T}\left(t, t^{\prime}\right) \int_{0}^{1} d \tau \rho_{q}^{\tau}\left(t^{\prime}\right) I_{G}^{a b}\left(\boldsymbol{r}, \boldsymbol{r}^{\prime} ; t^{\prime}\right) \rho_{q}^{1-\tau}\left(t^{\prime}\right) \beta \overleftrightarrow{\mu}_{b a}\left(\boldsymbol{r}, \boldsymbol{r}^{\prime} t^{\prime}\right) d t^{\prime} \\
& -\sum_{a b} \int d \boldsymbol{r} \int d \boldsymbol{r}^{\prime} \int_{-\infty}^{t} e^{\varepsilon\left(t^{\prime}-t\right)} \hat{T}\left(t, t^{\prime}\right) \int_{0}^{1} d \tau \rho_{q}^{\tau}\left(t^{\prime}\right) I_{g}^{a b}\left(\boldsymbol{r}, \boldsymbol{r}^{\prime} ; t^{\prime}\right) \rho_{q}^{1-\tau}\left(t^{\prime}\right) \beta \chi_{b a}\left(\boldsymbol{r}, \boldsymbol{r}^{\prime} t^{\prime}\right) d t^{\prime},
\end{aligned}
$$

where

$$
\begin{gathered}
I_{n}^{a}\left(\boldsymbol{r}^{\prime} ; t^{\prime}\right)=\left(1-P\left(t^{\prime}\right)\right) i \hat{L}\left(t^{\prime}\right) \hat{n}_{a}\left(\boldsymbol{r}^{\prime}\right), \quad I_{M}^{a}\left(\boldsymbol{r}^{\prime} ; t^{\prime}\right)=\left(1-P\left(t^{\prime}\right)\right) i \hat{L}\left(t^{\prime}\right) \hat{M}_{a}\left(\boldsymbol{r}^{\prime}\right), \\
I_{G}^{a b}\left(\boldsymbol{r}, \boldsymbol{r}^{\prime} ; t^{\prime}\right)=\left(1-P\left(t^{\prime}\right)\right) i \hat{L}\left(t^{\prime}\right) \stackrel{\leftrightarrow}{G}_{a b}\left(\boldsymbol{r}, \boldsymbol{r}^{\prime}\right), \quad I_{g}^{a b}\left(\boldsymbol{r}, \boldsymbol{r}^{\prime} ; t^{\prime}\right)=\left(1-P\left(t^{\prime}\right)\right) i \hat{L}\left(t^{\prime}\right) \hat{g}_{a b}\left(\boldsymbol{r}, \boldsymbol{r}^{\prime}\right)
\end{gathered}
$$

are the microscopic generalized fluxes describing diffusion, magneto-diffusion and reaction processes in the system and on which there are built the generalized transfer coefficients and functions of reactions between components that describe, in particular, oxidation stages $(1)-(4) . P\left(t^{\prime}\right)$ is the generalized Mori projection operator [35], which acts on dynamic variables (operators) and in this choice of abbreviated description parameters $\left\langle\hat{n}_{a}(\boldsymbol{r})\right\rangle^{t},\left\langle\boldsymbol{M}_{a}(\boldsymbol{r})\right\rangle^{t},\left\langle\hat{g}_{a b}\left(\boldsymbol{r}, \boldsymbol{r}^{\prime}\right)\right\rangle^{t},\left\langle\hat{\leftrightarrow}_{a b}\left(\boldsymbol{r}, \boldsymbol{r}^{\prime}\right)\right\rangle^{t}$ has the following form:

$$
\begin{aligned}
P\left(t^{\prime}\right) A(\boldsymbol{r})= & \langle A(\boldsymbol{r})\rangle_{q}^{t}+\sum_{a} \int d \boldsymbol{r}^{\prime} \frac{\delta\langle A(\boldsymbol{r})\rangle_{q}^{t}}{\delta\left\langle\hat{n}_{a}\left(\boldsymbol{r}^{\prime}\right)\right\rangle^{t}}\left(\hat{n}_{a}\left(\boldsymbol{r}^{\prime}\right)-\left\langle\hat{n}_{a}\left(\boldsymbol{r}^{\prime}\right)\right\rangle^{t}\right) \\
& +\sum_{a} \int d \boldsymbol{r}^{\prime} \frac{\delta\langle A(\boldsymbol{r})\rangle_{q}^{t}}{\delta\left\langle\hat{\boldsymbol{M}}_{a}\left(\boldsymbol{r}^{\prime}\right)\right\rangle^{t}}\left(\hat{\boldsymbol{M}}_{a}\left(\boldsymbol{r}^{\prime}\right)-\left\langle\hat{\boldsymbol{M}}_{a}\left(\boldsymbol{r}^{\prime}\right)\right\rangle^{t}\right) \\
& +\sum_{a b} \int d \boldsymbol{r}^{\prime} \int d \boldsymbol{r}^{\prime \prime} \frac{\delta\langle A(\boldsymbol{r})\rangle_{q}^{t}}{\delta\left\langle\hat{\leftrightarrow}_{a b}\left(\boldsymbol{r}^{\prime}, \boldsymbol{r}^{\prime \prime}\right)\right\rangle^{t}}\left(\hat{\leftrightarrow}_{a b}\left(\boldsymbol{r}^{\prime}, \boldsymbol{r}^{\prime \prime}\right)-\left\langle\hat{\leftrightarrow}_{a b}\left(\boldsymbol{r}^{\prime}, \boldsymbol{r}^{\prime \prime}\right)\right\rangle^{t}\right) \\
& +\sum_{a b} \int d \boldsymbol{r}^{\prime} \int d \boldsymbol{r}^{\prime \prime} \frac{\delta\langle A(\boldsymbol{r})\rangle_{q}^{t}}{\delta\left\langle\hat{g}_{a b}\left(\boldsymbol{r}^{\prime}, \boldsymbol{r}^{\prime \prime}\right)\right\rangle^{t}}\left(\hat{g}_{a b}\left(\boldsymbol{r}^{\prime}, \boldsymbol{r}^{\prime \prime}\right)-\left\langle\hat{g}_{a b}\left(\boldsymbol{r}^{\prime}, \boldsymbol{r}^{\prime \prime}\right)\right\rangle^{t}\right)
\end{aligned}
$$

and the following properties $P(t) P\left(t^{\prime}\right)=P(t), P(t)\left(1-P\left(t^{\prime}\right)\right)=0, P(t) \hat{n}_{a}\left(\boldsymbol{r}^{\prime}\right)=\hat{n}_{a}\left(\boldsymbol{r}^{\prime}\right), P(t) \hat{\boldsymbol{M}}_{a}\left(\boldsymbol{r}^{\prime}\right)=$ $\hat{\boldsymbol{M}}_{a}\left(\boldsymbol{r}^{\prime}\right), P(t) \stackrel{\hat{G}}{G}_{a b}\left(\boldsymbol{r}^{\prime}, \boldsymbol{r}^{\prime \prime}\right)=\stackrel{\leftrightarrow}{G}_{a b}\left(\boldsymbol{r}^{\prime}, \boldsymbol{r}^{\prime \prime}\right), P(t) \hat{g}_{a b}\left(\boldsymbol{r}^{\prime}, \boldsymbol{r}^{\prime \prime}\right)=\hat{g}_{a b}\left(\boldsymbol{r}^{\prime}, \boldsymbol{r}^{\prime \prime}\right)$.

Using the nonequilibrium statistical operator $\rho(t)$ (10) one can obtain a system of transfer equations. To find the system of transfer equations, we use the fact that:

$$
\frac{\partial}{\partial t}\langle\hat{X}\rangle^{t}=\langle\dot{\hat{X}}\rangle^{t}=\langle\dot{\hat{X}}\rangle_{q}^{t}+\langle(1-P(t)) \dot{\hat{X}}\rangle^{t}=\langle\dot{\hat{X}}\rangle_{q}^{t}+\left\langle I_{X}\right\rangle^{t}
$$

Mathematical Modeling and Computing, Vol. 8, No. 2, pp. 267-281 (2021) 
Using expression (10) for the nonequilibrium statistical operator, we obtain the transfer equation to describe the reaction-diffusion, magnetostrictive processes of magnetoactive particles that can be adsorbed on magnetoactive surface of the metal.

In expanded form, these transfer equations have the following structure:

$$
\begin{aligned}
& \frac{\partial}{\partial t}\left\langle\hat{n}_{a}(\boldsymbol{r})\right\rangle^{t}=-\sum_{b} \int d \boldsymbol{r}^{\prime} \int_{-\infty}^{t} e^{\varepsilon\left(t^{\prime}-t\right)} \frac{\partial}{\partial \boldsymbol{r}} D_{n n}^{a b}\left(\boldsymbol{r}, \boldsymbol{r}^{\prime} ; t, t^{\prime}\right) \frac{\partial}{\partial \boldsymbol{r}^{\prime}} \beta \mu_{b}\left(\boldsymbol{r}^{\prime}, t^{\prime}\right) d t^{\prime} \\
& -\sum_{b} \int d \boldsymbol{r}^{\prime} \int_{-\infty}^{t} e^{\varepsilon\left(t^{\prime}-t\right)} \frac{\partial}{\partial \boldsymbol{r}} D_{n R}^{a b}\left(\boldsymbol{r}, \boldsymbol{r}^{\prime} ; t, t^{\prime}\right) \frac{\partial}{\partial \boldsymbol{r}^{\prime}} \beta \boldsymbol{b}_{b}\left(\boldsymbol{r}^{\prime}, t^{\prime}\right) d t^{\prime} \\
& -\sum_{b} \int d \boldsymbol{r}^{\prime} \int_{-\infty}^{t} e^{\varepsilon\left(t^{\prime}-t\right)} \frac{\partial}{\partial \boldsymbol{r}} D_{n M}^{a b}\left(\boldsymbol{r}, \boldsymbol{r}^{\prime} ; t, t^{\prime}\right) \beta\left(\boldsymbol{b}_{b}\left(\boldsymbol{r}^{\prime}, t^{\prime}\right)-\boldsymbol{B}\left(\boldsymbol{r}^{\prime}, t^{\prime}\right)\right) d t^{\prime} \\
& +\sum_{b c} \int d \boldsymbol{r}^{\prime} \int d \boldsymbol{r}^{\prime \prime} \int_{-\infty}^{t} e^{\varepsilon\left(t^{\prime}-t\right)} \frac{\partial}{\partial \boldsymbol{r}} K_{n G}^{a b c}\left(\boldsymbol{r}, \boldsymbol{r}^{\prime}, \boldsymbol{r}^{\prime \prime} ; t, t^{\prime}\right) \beta \stackrel{\leftrightarrow}{\mu}_{c b}\left(\boldsymbol{r}^{\prime}, \boldsymbol{r}^{\prime \prime}, t^{\prime}\right) d t^{\prime} \\
& +\sum_{b c} \int d \boldsymbol{r}^{\prime} \int d \boldsymbol{r}^{\prime \prime} \int_{-\infty}^{t} e^{\varepsilon\left(t^{\prime}-t\right)} \frac{\partial}{\partial \boldsymbol{r}} K_{n g}^{a b c}\left(\boldsymbol{r}, \boldsymbol{r}^{\prime}, \boldsymbol{r}^{\prime \prime} ; t, t^{\prime}\right) \beta \chi_{c b}\left(\boldsymbol{r}^{\prime}, \boldsymbol{r}^{\prime \prime}, t^{\prime}\right) d t^{\prime} ; \\
& \frac{\partial}{\partial t}\left\langle\hat{\boldsymbol{M}}_{a}(\boldsymbol{r})\right\rangle^{t}=\sum_{b} \int d \boldsymbol{r}^{\prime}\left\langle\frac{i}{h}\left[\hat{\boldsymbol{M}}_{a}(\boldsymbol{r}), \hat{\boldsymbol{M}}_{b}\left(\boldsymbol{r}^{\prime}\right)\right]\right\rangle_{q}^{t}\left(\boldsymbol{b}_{b}\left(\boldsymbol{r}^{\prime}, t\right)-\boldsymbol{B}\left(\boldsymbol{r}^{\prime}, t\right)\right) \\
& -\sum_{b} \int d \boldsymbol{r}^{\prime} \int_{-\infty}^{t} e^{\varepsilon\left(t^{\prime}-t\right)} D_{M n}^{a b}\left(\boldsymbol{r}, \boldsymbol{r}^{\prime} ; t, t^{\prime}\right) \frac{\partial}{\partial \boldsymbol{r}^{\prime}} \beta \mu_{b}\left(\boldsymbol{r}^{\prime}, t^{\prime}\right) d t^{\prime} \\
& -\sum_{b} \int d \boldsymbol{r}^{\prime} \int_{-\infty}^{t} e^{\varepsilon\left(t^{\prime}-t\right)} D_{M M}^{a b}\left(\boldsymbol{r}, \boldsymbol{r}^{\prime} ; t, t^{\prime}\right) \beta\left(\boldsymbol{b}_{b}\left(\boldsymbol{r}^{\prime}, t^{\prime}\right)-\boldsymbol{B}\left(\boldsymbol{r}^{\prime}, t^{\prime}\right)\right) d t^{\prime} \\
& -\sum_{b c} \int d \boldsymbol{r}^{\prime} \int d \boldsymbol{r}^{\prime \prime} \int_{-\infty}^{t} e^{\varepsilon\left(t^{\prime}-t\right)} K_{M G}^{a b c}\left(\boldsymbol{r}, \boldsymbol{r}^{\prime}, \boldsymbol{r}^{\prime \prime} ; t, t^{\prime}\right) \beta \overleftrightarrow{\mu}_{c b}\left(\boldsymbol{r}^{\prime}, \boldsymbol{r}^{\prime \prime}, t^{\prime}\right) d t^{\prime} \\
& -\sum_{b c} \int d \boldsymbol{r}^{\prime} \int d \boldsymbol{r}^{\prime \prime} \int_{-\infty}^{t} e^{\varepsilon\left(t^{\prime}-t\right)} \frac{\partial}{\partial \boldsymbol{r}} K_{M g}^{a b c}\left(\boldsymbol{r}, \boldsymbol{r}^{\prime}, \boldsymbol{r}^{\prime \prime} ; t, t^{\prime}\right) \beta \chi_{c b}\left(\boldsymbol{r}^{\prime}, \boldsymbol{r}^{\prime \prime}, t^{\prime}\right) d t^{\prime} ; \\
& \frac{\partial}{\partial t}\left\langle\hat{\leftrightarrow}_{a b}\left(\boldsymbol{r}, \boldsymbol{r}^{\prime}\right)\right\rangle^{t}=\left\langle i \hat{L}(t) \hat{\leftrightarrow}_{a b}\left(\boldsymbol{r}, \boldsymbol{r}^{\prime}\right)\right\rangle_{q}^{t}+\sum_{c} \int d \boldsymbol{r}^{\prime \prime} \int_{-\infty}^{t} e^{\varepsilon\left(t^{\prime}-t\right)} K_{G n}^{a b c}\left(\boldsymbol{r}, \boldsymbol{r}^{\prime}, \boldsymbol{r}^{\prime \prime} ; t, t^{\prime}\right) \frac{\partial}{\partial \boldsymbol{r}^{\prime \prime}} \beta \mu_{c}\left(\boldsymbol{r}^{\prime \prime}, t^{\prime}\right) d t^{\prime} \\
& +\sum_{c} \int d \boldsymbol{r}^{\prime \prime} \int_{-\infty}^{t} e^{\varepsilon\left(t^{\prime}-t\right)} K_{G R}^{a b c}\left(\boldsymbol{r}, \boldsymbol{r}^{\prime}, \boldsymbol{r}^{\prime \prime} ; t, t^{\prime}\right) \frac{\partial}{\partial \boldsymbol{r}^{\prime \prime}} \beta \boldsymbol{b}_{c}\left(\boldsymbol{r}^{\prime \prime}, t^{\prime}\right) d t^{\prime} \\
& -\sum_{c} \int d \boldsymbol{r}^{\prime \prime} \int_{-\infty}^{t} e^{\varepsilon\left(t^{\prime}-t\right)} K_{G M}^{a b c}\left(\boldsymbol{r}, \boldsymbol{r}^{\prime}, \boldsymbol{r}^{\prime \prime} ; t, t^{\prime}\right) \beta\left(\boldsymbol{b}_{c}\left(\boldsymbol{r}^{\prime \prime}, t^{\prime}\right)-\boldsymbol{B}\left(\boldsymbol{r}^{\prime \prime}, t^{\prime}\right)\right) d t^{\prime} \\
& -\sum_{d c} \int d \boldsymbol{r}^{\prime \prime} \int d \boldsymbol{r}^{\prime \prime \prime} \int_{-\infty}^{t} e^{\varepsilon\left(t^{\prime}-t\right)} K_{G G}^{a b c d}\left(\boldsymbol{r}, \boldsymbol{r}^{\prime}, \boldsymbol{r}^{\prime \prime}, \boldsymbol{r}^{\prime \prime \prime} ; t, t^{\prime}\right) \beta \overleftrightarrow{\mu}_{d b}\left(\boldsymbol{r}^{\prime \prime}, \boldsymbol{r}^{\prime \prime \prime}, t^{\prime}\right) d t^{\prime} \\
& -\sum_{d c} \int d \boldsymbol{r}^{\prime \prime} \int d \boldsymbol{r}^{\prime \prime \prime} \int_{-\infty}^{t} e^{\varepsilon\left(t^{\prime}-t\right)} K_{G g}^{a b c d}\left(\boldsymbol{r}, \boldsymbol{r}^{\prime}, \boldsymbol{r}^{\prime \prime}, \boldsymbol{r}^{\prime \prime \prime} ; t, t^{\prime}\right) \beta \chi_{d c}\left(\boldsymbol{r}^{\prime \prime}, \boldsymbol{r}^{\prime \prime \prime}, t^{\prime}\right) d t^{\prime} ; \\
& \frac{\partial}{\partial t}\left\langle\hat{g}_{a b}\left(\boldsymbol{r}, \boldsymbol{r}^{\prime}\right)\right\rangle^{t}=\left\langle i \hat{L}\left(t^{\prime}\right) \hat{g}_{a b}\left(\boldsymbol{r}, \boldsymbol{r}^{\prime}\right)\right\rangle_{q}^{t}+\sum_{c} \int d \boldsymbol{r}^{\prime \prime} \int_{-\infty}^{t} e^{\varepsilon\left(t^{\prime}-t\right)} K_{g n}^{a b c}\left(\boldsymbol{r}, \boldsymbol{r}^{\prime}, \boldsymbol{r}^{\prime \prime} ; t, t^{\prime}\right) \frac{\partial}{\partial \boldsymbol{r}^{\prime \prime}} \beta \mu_{c}\left(\boldsymbol{r}^{\prime \prime}, t^{\prime}\right) d t^{\prime}
\end{aligned}
$$




$$
\begin{aligned}
& +\sum_{c} \int d \boldsymbol{r}^{\prime \prime} \int_{-\infty}^{t} e^{\varepsilon\left(t^{\prime}-t\right)} K_{g R}^{a b c}\left(\boldsymbol{r}, \boldsymbol{r}^{\prime}, \boldsymbol{r}^{\prime \prime} ; t, t^{\prime}\right) \frac{\partial}{\partial \boldsymbol{r}^{\prime \prime}} \beta \boldsymbol{b}_{c}\left(\boldsymbol{r}^{\prime \prime}, t^{\prime}\right) d t^{\prime} \\
& -\sum_{c} \int d \boldsymbol{r}^{\prime \prime} \int_{-\infty}^{t} e^{\varepsilon\left(t^{\prime}-t\right)} K_{g M}^{a b c}\left(\boldsymbol{r}, \boldsymbol{r}^{\prime}, \boldsymbol{r}^{\prime \prime} ; t, t^{\prime}\right) \beta\left(\boldsymbol{b}_{c}\left(\boldsymbol{r}^{\prime \prime}, t^{\prime}\right)-\boldsymbol{B}\left(\boldsymbol{r}^{\prime \prime}, t^{\prime}\right)\right) d t^{\prime} \\
& -\sum_{d c} \int d \boldsymbol{r}^{\prime \prime} \int d \boldsymbol{r}^{\prime \prime \prime} \int_{-\infty}^{t} e^{\varepsilon\left(t^{\prime}-t\right)} K_{g G}^{a b c d}\left(\boldsymbol{r}, \boldsymbol{r}^{\prime}, \boldsymbol{r}^{\prime \prime}, \boldsymbol{r}^{\prime \prime \prime} ; t, t^{\prime}\right) \beta \stackrel{\leftrightarrow}{\mu}_{d b}\left(\boldsymbol{r}^{\prime \prime}, \boldsymbol{r}^{\prime \prime \prime}, t^{\prime}\right) d t^{\prime} \\
& -\sum_{d c} \int d \boldsymbol{r}^{\prime \prime} \int d \boldsymbol{r}^{\prime \prime \prime} \int_{-\infty}^{t} e^{\varepsilon\left(t^{\prime}-t\right)} K_{g g}^{a b c d}\left(\boldsymbol{r}, \boldsymbol{r}^{\prime}, \boldsymbol{r}^{\prime \prime}, \boldsymbol{r}^{\prime \prime \prime} ; t, t^{\prime}\right) \beta \chi_{d c}\left(\boldsymbol{r}^{\prime \prime}, \boldsymbol{r}^{\prime \prime \prime}, t^{\prime}\right) d t^{\prime}
\end{aligned}
$$

Here

$$
D_{n n}^{a b}\left(\boldsymbol{r}, \boldsymbol{r}^{\prime} ; t, t^{\prime}\right)=\operatorname{Sp}\left((1-P(t)) \frac{1}{m_{a}} \hat{\boldsymbol{p}}_{a}(\boldsymbol{r}) T\left(t, t^{\prime}\right) \int_{0}^{1} d \tau \rho_{q}^{\tau}\left(t^{\prime}\right)\left(1-P\left(t^{\prime}\right)\right) \frac{1}{m_{b}} \hat{\boldsymbol{p}}_{b}\left(\boldsymbol{r}^{\prime}\right) \rho_{q}^{1-\tau}\left(t^{\prime}\right)\right)
$$

is the generalized diffusion coefficient of magnetoactive particles, which is written in the form of GreenKubo [35], $\hat{\boldsymbol{p}}_{a}(\boldsymbol{r})=\sum_{j=1}^{N_{a}} \boldsymbol{p}_{j} \delta\left(\boldsymbol{r}^{\prime}-\boldsymbol{r}_{j}\right)$ is the microscopic moment density of magnetoactive particles of sort $a$;

$$
D_{n R}^{a b}\left(\boldsymbol{r}, \boldsymbol{r}^{\prime} ; t, t^{\prime}\right)=\operatorname{Sp}\left((1-P(t)) \frac{1}{m_{a}} \hat{\boldsymbol{p}}_{a}(\boldsymbol{r}) T\left(t, t^{\prime}\right) \int_{0}^{1} d \tau \rho_{q}^{\tau}\left(t^{\prime}\right)\left(1-P\left(t^{\prime}\right)\right) R_{b}\left(\boldsymbol{r}^{\prime}\right) \rho_{q}^{1-\tau}\left(t^{\prime}\right)\right)
$$

is the generalized transfer coefficient describing magnetostrictive processes, where

$$
\begin{gathered}
R_{b}\left(\boldsymbol{r}^{\prime}\right)=\sum_{j=1}^{N_{b}} \frac{\boldsymbol{p}_{j} \mu_{b} \boldsymbol{S}_{j}}{m_{b}} \delta\left(\boldsymbol{r}^{\prime}-\boldsymbol{r}_{j}\right) . \\
D_{M M}^{a b}\left(\boldsymbol{r}, \boldsymbol{r}^{\prime} ; t, t^{\prime}\right)=\operatorname{Sp}\left((1-P(t)) i \hat{L}(t) \hat{\boldsymbol{S}}_{a}(\boldsymbol{r}) T\left(t, t^{\prime}\right) \int_{0}^{1} d \tau \rho_{q}^{\tau}\left(t^{\prime}\right)\left(1-P\left(t^{\prime}\right)\right) i \hat{L}(t) \hat{\boldsymbol{S}}_{b}\left(\boldsymbol{r}^{\prime}\right) \rho_{q}^{1-\tau}\left(t^{\prime}\right)\right)
\end{gathered}
$$

is the generalized magnetic diffusion coefficient of magnetoactive particles of sort $a$ and $b$. The generalized transfer coefficient $D_{M n}^{a b}\left(\boldsymbol{r}, \boldsymbol{r}^{\prime} ; t, t^{\prime}\right)$ as well as $D_{n R}^{a b}\left(\boldsymbol{r}, \boldsymbol{r}^{\prime} ; t, t^{\prime}\right)$ describe the magnetostrictive processes. The generalized transfer kernels $K_{G n}^{a b c}\left(\boldsymbol{r}, \boldsymbol{r}^{\prime}, \boldsymbol{r}^{\prime \prime} ; t, t^{\prime}\right), K_{G M}^{a b c}\left(\boldsymbol{r}, \boldsymbol{r}^{\prime}, \boldsymbol{r}^{\prime \prime} ; t, t^{\prime}\right)$, $K_{G G}^{a b c d}\left(\boldsymbol{r}, \boldsymbol{r}^{\prime}, \boldsymbol{r}^{\prime \prime}, \boldsymbol{r}^{\prime \prime \prime} ; t, t^{\prime}\right), K_{G g}^{a b c d}\left(\boldsymbol{r}, \boldsymbol{r}^{\prime}, \boldsymbol{r}^{\prime \prime} \boldsymbol{r}^{\prime \prime \prime} ; t, t^{\prime}\right), K_{g g}^{a b c d}\left(\boldsymbol{r}, \boldsymbol{r}^{\prime}, \boldsymbol{r}^{\prime \prime} \boldsymbol{r}^{\prime \prime \prime} ; t, t^{\prime}\right)$ describe reaction-diffusion processes taking into account magnetic interaction and are correlation functions of the third and fourth order in terms of dynamic variables. In particular, function $K_{G G}^{a b c d}\left(\boldsymbol{r}, \boldsymbol{r}^{\prime}, \boldsymbol{r}^{\prime \prime}, \boldsymbol{r}^{\prime \prime \prime} ; t, t^{\prime}\right)$ has the form:

$$
\begin{aligned}
K_{G G}^{a b c d}\left(\boldsymbol{r}, \boldsymbol{r}^{\prime}, \boldsymbol{r}^{\prime \prime}, \boldsymbol{r}^{\prime \prime \prime} ; t, t^{\prime}\right)=\operatorname{Sp}\left((1-P(t)) i \hat{L}(t) \stackrel{\hat{\leftrightarrow}}{G} a b\left(\boldsymbol{r}, \boldsymbol{r}^{\prime}\right) T\left(t, t^{\prime}\right) \int_{0}^{1} d \tau \rho_{q}^{\tau}\left(t^{\prime}\right)\right. \\
\left.\times\left(1-P\left(t^{\prime}\right)\right) i \hat{L}(t) \stackrel{\leftrightarrow}{G}_{c d}\left(\boldsymbol{r}^{\prime \prime}, \boldsymbol{r}^{\prime \prime \prime}\right) \rho_{q}^{1-\tau}\left(t^{\prime}\right)\right) .
\end{aligned}
$$

The obtained here transfer coefficients and transfer kernels describe dissipative correlations in the system of magnetoactive particles (molecules, atoms, anions, electrons), which are in the inhomogeneous magnetic field of magnetoactive surface of the metal. They have a complex structure and describe the non-Markov, nonlinear diffusion, reaction-diffusion, magnetostrictive and spindiffusion processes. The influence of the magnetoactive surface on these processes is taken into account both through the magnetic field $\boldsymbol{B}(\boldsymbol{r} ; t)$ and through the magnetic interaction and adsorption potential included in the Hamiltonian of the system. The obtained transfer equations (13)-(16) can describe diffusion, magnetostrictive and spindiffusion processes also in the presence of external magnetic fields. 


\section{Reaction-diffusion transfer equations for weakly nonequilibrium catalytic processes}

We consider the reaction-diffusion processes characterized by small fluctuations of nonequilibrium thermodynamic parameters $\boldsymbol{F}_{\nu}(\boldsymbol{r} ; t)$ and $\boldsymbol{F}_{\eta}\left(\boldsymbol{r}, \boldsymbol{r}^{\prime} ; t\right)$

$$
\begin{gathered}
\boldsymbol{F}_{\nu}(\boldsymbol{r} ; t)=\left(\beta \mu_{a}(\boldsymbol{r} ; t), \beta \boldsymbol{b}_{a}(\boldsymbol{r} ; t)\right)^{\mathrm{T}}, \\
\boldsymbol{F}_{\eta}\left(\boldsymbol{r}, \boldsymbol{r}^{\prime} ; t\right)=\left(\beta \overleftrightarrow{\mu}_{a b}\left(\boldsymbol{r}, \boldsymbol{r}^{\prime} ; t\right), \beta \chi_{a b}\left(\boldsymbol{r}, \boldsymbol{r}^{\prime} ; t\right)\right)^{\mathrm{T}} .
\end{gathered}
$$

from their locally equilibrium values $\boldsymbol{F}_{\nu}(\boldsymbol{r})$ and $\boldsymbol{F}_{\eta}\left(\boldsymbol{r}, \boldsymbol{r}^{\prime}\right)$. In this case, we decompose the relevant statistical operator $\rho_{q}(t)$ by fluctuations $\delta \boldsymbol{F}_{\nu}(\boldsymbol{r} ; t)=\boldsymbol{F}_{\nu}(\boldsymbol{r} ; t)-\boldsymbol{F}_{\nu}(\boldsymbol{r})$ and $\delta \boldsymbol{F}_{\eta}\left(\boldsymbol{r}, \boldsymbol{r}^{\prime} ; t\right)=\boldsymbol{F}_{\eta}\left(\boldsymbol{r}, \boldsymbol{r}^{\prime} ; t\right)-$ $\boldsymbol{F}_{\eta}\left(\boldsymbol{r}, \boldsymbol{r}^{\prime}\right)$ into a series and limit ourselves to a linear approximation by these fluctuations. In this approximation, the fluctuations of nonequilibrium thermodynamic parameters $\delta \boldsymbol{F}_{\nu}(\boldsymbol{r} ; t), \delta \boldsymbol{F}_{\eta}\left(\boldsymbol{r}, \boldsymbol{r}^{\prime} ; t\right)$ are determined from the corresponding self-agreement conditions (9). As a result, for the relevant statistical operator we obtain in this approximation:

$$
\begin{aligned}
\rho_{q}^{0}(t)= & \rho_{0}\left(1+\sum_{a b} \iint\left\langle\delta \hat{n}_{a}(\boldsymbol{r})\right\rangle^{t}\left[\tilde{\Phi}_{n n}^{-1}\left(\boldsymbol{r}, \boldsymbol{r}^{\prime}\right)\right]_{a b} \hat{n}_{b}\left(\boldsymbol{r}^{\prime} ; \tau\right) d \boldsymbol{r} d \boldsymbol{r}^{\prime}\right. \\
& +\sum_{a b} \iint\left\langle\delta \overline{\boldsymbol{M}}_{a}(\boldsymbol{r})\right\rangle^{t}\left[\tilde{\Phi}_{\bar{M} \bar{M}}^{-1}\left(\boldsymbol{r}, \boldsymbol{r}^{\prime}\right)\right]_{a b} \overline{\boldsymbol{M}}_{b}\left(\boldsymbol{r}^{\prime} ; \tau\right) d \boldsymbol{r} d \boldsymbol{r}^{\prime} \\
& +\sum_{a b c d} \iiint \int\left\langle\delta \bar{g}_{a b}\left(\boldsymbol{r}, \boldsymbol{r}^{\prime}\right)\right\rangle^{t}\left[\tilde{\Phi}_{\bar{g} \bar{g}}^{-1}\left(\boldsymbol{r}, \boldsymbol{r}^{\prime} ; \boldsymbol{r}^{\prime \prime}, \boldsymbol{r}^{\prime \prime \prime}\right)\right]_{a b c d} \bar{g}_{c d}\left(\boldsymbol{r}^{\prime \prime}, \boldsymbol{r}^{\prime \prime \prime} ; \tau\right) d \boldsymbol{r} d \boldsymbol{r}^{\prime} d \boldsymbol{r}^{\prime \prime} d \boldsymbol{r}^{\prime \prime \prime} \\
& \left.+\sum_{a b c d} \iiint \int\left\langle\delta \stackrel{\leftrightarrow}{G}_{a b}\left(\boldsymbol{r}, \boldsymbol{r}^{\prime}\right)\right\rangle^{t}\left[\tilde{\Phi}_{\bar{G} \bar{G}}^{-1}\left(\boldsymbol{r}, \boldsymbol{r}^{\prime} ; \boldsymbol{r}^{\prime \prime}, \boldsymbol{r}^{\prime \prime \prime}\right)\right]_{a b c d} \stackrel{\leftrightarrow}{G}_{c d}\left(\boldsymbol{r}^{\prime \prime}, \boldsymbol{r}^{\prime \prime \prime} ; \tau\right) d \boldsymbol{r} d \boldsymbol{r}^{\prime} d \boldsymbol{r}^{\prime \prime} d \boldsymbol{r}^{\prime \prime \prime}\right),
\end{aligned}
$$

where

$$
\begin{aligned}
\rho_{0}= & \exp \left\{-\Phi-\beta\left(\hat{H}-\sum_{a} \int \mu_{a}(\boldsymbol{r}) \hat{n}_{a}(\boldsymbol{r}) d \boldsymbol{r}-\sum_{a b} \int d \boldsymbol{r} \int d \boldsymbol{r}^{\prime} \overleftrightarrow{\mu}_{b a}(\boldsymbol{r}, \boldsymbol{r}) \stackrel{\leftrightarrow}{G}_{a b}\left(\boldsymbol{r}, \boldsymbol{r}^{\prime}\right)\right.\right. \\
& \left.-\sum_{a} \int d \boldsymbol{r} \hat{\boldsymbol{M}}_{a}(\boldsymbol{r}) \boldsymbol{b}_{a}(\boldsymbol{r})-\sum_{a b} \int d \boldsymbol{r} \int d \boldsymbol{r}^{\prime} \chi_{b a}\left(\boldsymbol{r}, \boldsymbol{r}^{\prime}\right) \hat{g}_{a b}\left(\boldsymbol{r}, \boldsymbol{r}^{\prime}\right)-\mu_{m} N_{m}\right\}
\end{aligned}
$$

is the equilibrium statistical operator of the system. $A(\boldsymbol{r} ; \tau)=\int_{0}^{1} d \tau \rho_{0}^{\tau} A(\boldsymbol{r}) \rho_{0}^{-\tau}$. In this approximation, the fluctuations of parameters in the abbreviated description of nonequilibrium processes have the following structure: $\delta \hat{n}_{a}(\boldsymbol{r})=\hat{n}_{a}(\boldsymbol{r})-\left\langle\hat{n}_{a}(\boldsymbol{r})\right\rangle_{0}, \delta \overline{\boldsymbol{M}}_{a}(\boldsymbol{r})=\overline{\boldsymbol{M}}_{a}(\boldsymbol{r})-\left\langle\overline{\boldsymbol{M}}_{a}(\boldsymbol{r})\right\rangle_{0}$, $\delta \bar{g}_{a b}\left(\boldsymbol{r}, \boldsymbol{r}^{\prime}\right)=\bar{g}_{a b}\left(\boldsymbol{r}, \boldsymbol{r}^{\prime}\right)-\left\langle\bar{g}_{a b}\left(\boldsymbol{r}, \boldsymbol{r}^{\prime}\right)\right\rangle_{0}, \delta \stackrel{\overleftrightarrow{G}}{G}_{a b}\left(\boldsymbol{r}, \boldsymbol{r}^{\prime}\right)=\stackrel{\leftrightarrow}{G}_{a b}\left(\boldsymbol{r}, \boldsymbol{r}^{\prime}\right)-\left\langle\stackrel{\leftrightarrow}{G}_{a b}\left(\boldsymbol{r}, \boldsymbol{r}^{\prime}\right)\right\rangle_{0},\left(\langle\ldots\rangle_{0}=\mathrm{Sp}\left[\ldots \rho_{0}\right]\right.$ is the averaging with the equilibrium statistical operator $\left.\rho_{0}\right)$ in which $\overline{\boldsymbol{M}}_{a}(\boldsymbol{r}), \bar{g}_{a b}\left(\boldsymbol{r}, \boldsymbol{r}^{\prime}\right), \stackrel{\vec{G}}{G}_{a b}\left(\boldsymbol{r}, \boldsymbol{r}^{\prime}\right)$ are the new description parameters, that arose by excluding nonequilibrium parameters $\delta \boldsymbol{F}_{\nu}(\boldsymbol{r} ; t), \delta \boldsymbol{F}_{\eta}\left(\boldsymbol{r}, \boldsymbol{r}^{\prime} ; t\right)$ from the corresponding self-agreement conditions (9):

$$
\overline{\boldsymbol{M}}_{a}(\boldsymbol{r})=\left(1-P_{n n}\right) \hat{\boldsymbol{M}}_{a}(\boldsymbol{r}),
$$

where $P_{n n}$ is the Mori-type projection operator built on variables $\hat{n}_{b}\left(\boldsymbol{r}^{\prime}\right)$

$$
\begin{gathered}
P_{n n}(\ldots)=\sum_{b c} \iint\left\langle\ldots \hat{n}_{b}\left(\boldsymbol{r}^{\prime}\right)\right\rangle_{0}\left[\tilde{\Phi}_{n n}^{-1}\left(\boldsymbol{r}^{\prime}, \boldsymbol{r}^{\prime \prime}\right)\right]_{b c} \hat{n}_{c}\left(\boldsymbol{r}^{\prime \prime} ; \tau\right) d \boldsymbol{r}^{\prime} d \boldsymbol{r}^{\prime \prime} \\
\bar{g}_{a b}\left(\boldsymbol{r}, \boldsymbol{r}^{\prime}\right)=\left(1-P_{n n}-P_{\bar{M} \bar{M}}\right) \hat{g}_{a b}\left(\boldsymbol{r}, \boldsymbol{r}^{\prime}\right),
\end{gathered}
$$

and $P_{\bar{M} \bar{M}}$ is the Mori-type projection operator built on variables $\overline{\boldsymbol{M}}_{c}\left(\boldsymbol{r}^{\prime \prime}\right)$

$$
\begin{aligned}
P_{\bar{M} \bar{M}}(\ldots)= & -\sum_{c d} \iint\left\langle\ldots \bar{M}_{c}\left(\boldsymbol{r}^{\prime \prime}\right)\right\rangle_{0}\left[\tilde{\Phi}_{\bar{M} \bar{M}}^{-1}\left(\boldsymbol{r}^{\prime \prime}, \boldsymbol{r}^{\prime \prime \prime}\right)\right]_{c d} \overline{\boldsymbol{M}}_{d}\left(\boldsymbol{r}^{\prime \prime \prime} ; \tau\right) d \boldsymbol{r}^{\prime \prime} d \boldsymbol{r}^{\prime \prime \prime}, \\
& \stackrel{\leftrightarrow}{G}_{a b}\left(\boldsymbol{r}, \boldsymbol{r}^{\prime}\right)=\left(1-P_{n n}-P_{\bar{M} \bar{M}}-P_{\bar{g} \bar{g}} \hat{\leftrightarrow}_{a b}\left(\boldsymbol{r}, \boldsymbol{r}^{\prime}\right),\right.
\end{aligned}
$$

Mathematical Modeling and Computing, Vol.8, No. 2, pp. 267-281 (2021) 
and $P_{\bar{g} \bar{g}}$ is the Mori-type projection operator built on variables $\bar{g}_{c d}\left(\boldsymbol{r}^{\prime \prime}, \boldsymbol{r}^{\prime \prime \prime}\right)$

$$
P_{\bar{g} \bar{g}}(\ldots)=\sum_{c d c^{\prime} d^{\prime}} \iiint \int\left\langle\ldots \bar{g}_{c d}\left(\boldsymbol{r}^{\prime \prime}, \boldsymbol{r}^{\prime \prime \prime}\right)\right\rangle_{0}\left[\tilde{\Phi}_{\bar{g} \bar{g}}^{-1}\left(\boldsymbol{r}^{\prime \prime}, \boldsymbol{r}^{\prime \prime \prime \prime} ; \boldsymbol{r}^{\prime \prime \prime \prime}, \boldsymbol{r}^{\prime \prime \prime \prime \prime}\right)\right]_{c d c^{\prime} d^{\prime}} \bar{g}_{c^{\prime} d^{\prime}}\left(\boldsymbol{r}^{\prime \prime \prime \prime \prime}, \boldsymbol{r}^{\prime \prime \prime \prime \prime \prime} ; \tau\right) d \boldsymbol{r}^{\prime \prime} d \boldsymbol{r}^{\prime \prime \prime} d \boldsymbol{r}^{\prime \prime \prime \prime \prime} d \boldsymbol{r}^{\prime \prime \prime \prime \prime}
$$

In the structure of projection operators $P_{n n}, P_{\bar{M} \bar{M}}, P_{\bar{g} \bar{g}}$ the corresponding functions $\left[\tilde{\Phi}_{n n}^{-1}\left(\boldsymbol{r}^{\prime}, \boldsymbol{r}^{\prime \prime}\right)\right]_{b c}$ are the elements of matrix inverse to the matrix, elements of which are $\Phi_{n n}^{c a}\left(\boldsymbol{r}^{\prime \prime}, \boldsymbol{r}\right)=\left\langle\hat{n}_{c}\left(\boldsymbol{r}^{\prime \prime}\right) \hat{n}_{a}(\boldsymbol{r} ; \tau)\right\rangle_{0}=$ $\left\langle\hat{g}_{c a}\left(\boldsymbol{r}^{\prime \prime}, \boldsymbol{r}\right)\right\rangle_{0}$ - the equilibrium pair structural distribution functions of the corresponding particles. Functions $\left[\tilde{\Phi}_{n n}^{-1}\left(\boldsymbol{r}^{\prime}, \boldsymbol{r}^{\prime \prime}\right)\right]_{b c}$ are found from integral relations

$$
\sum_{c} \int\left[\tilde{\Phi}_{n n}^{-1}\left(\boldsymbol{r}^{\prime}, \boldsymbol{r}^{\prime \prime}\right)\right]_{b c} \Phi_{n n}^{c a}\left(\boldsymbol{r}^{\prime \prime}, \boldsymbol{r}\right) d \boldsymbol{r}^{\prime \prime}=\delta_{b a} \delta\left(\boldsymbol{r}^{\prime}-\boldsymbol{r}\right)
$$

Functions $\left[\tilde{\Phi}_{\bar{M} \bar{M}}^{-1}\left(\boldsymbol{r}^{\prime}, \boldsymbol{r}^{\prime \prime}\right)\right]_{b c}$ are the elements of matrix inverse to the matrix, elements of which are $\Phi_{\bar{M} \bar{M}}^{c a}\left(\boldsymbol{r}^{\prime \prime}, \boldsymbol{r}\right)$ - the equilibrium correlation functions that describe the magnetic, magnetostrictive and structural properties of particles in the system. $\left[\tilde{\Phi}_{\bar{M} \bar{M}}^{-1}\left(\boldsymbol{r}^{\prime}, \boldsymbol{r}^{\prime \prime}\right)\right]_{b c}$ are found from integral relations

$$
\sum_{c} \int\left[\tilde{\Phi}_{\bar{M} \bar{M}}^{-1}\left(\boldsymbol{r}^{\prime}, \boldsymbol{r}^{\prime \prime}\right)\right]_{b c} \Phi_{\bar{M} \bar{M}}^{c a}\left(\boldsymbol{r}^{\prime \prime}, \boldsymbol{r}\right) d \boldsymbol{r}^{\prime \prime}=\delta_{b a} \delta\left(\boldsymbol{r}^{\prime}-\boldsymbol{r}\right)
$$

with the correlation function $\Phi_{\bar{M}}^{c a}\left(\boldsymbol{r}^{\prime \prime}, \boldsymbol{r}\right)$ in the following form:

$$
\begin{aligned}
\Phi_{\bar{M}}^{c a}\left(\boldsymbol{r}^{\prime \prime}, \boldsymbol{r}\right)= & \left\langle\left(1-P_{n n}\right) \hat{\boldsymbol{M}}_{c}\left(\boldsymbol{r}^{\prime \prime}\right)\left(1-P_{n n}\right) \hat{\boldsymbol{M}}_{a}(\boldsymbol{r} ; \tau)\right\rangle_{0} \\
= & \Phi_{M M}^{c a}\left(\boldsymbol{r}^{\prime \prime}, \boldsymbol{r}\right)-\sum_{b^{\prime} c^{\prime}} \iint \Phi_{M n}^{c b^{\prime}}\left(\boldsymbol{r}^{\prime \prime}, \boldsymbol{r}^{\prime \prime \prime}\right)\left[\tilde{\Phi}_{n n}^{-1}\left(\boldsymbol{r}^{\prime \prime \prime}, \boldsymbol{r}^{\prime \prime \prime \prime}\right)\right]_{b^{\prime} c^{\prime}} \Phi_{n M}^{c^{\prime} a}\left(\boldsymbol{r}^{\prime \prime \prime \prime}, \boldsymbol{r}\right) d \boldsymbol{r}^{\prime \prime \prime} d \boldsymbol{r}^{\prime \prime \prime \prime} \\
& -\sum_{d^{\prime} k^{\prime}} \iint \Phi_{M n}^{a d^{\prime}}\left(\boldsymbol{r}, \boldsymbol{r}^{\prime \prime \prime \prime \prime}\right)\left[\tilde{\Phi}_{n n}^{-1}\left(\boldsymbol{r}^{\prime \prime \prime \prime \prime \prime}, \boldsymbol{r}^{\prime \prime \prime \prime \prime \prime \prime \prime}\right)\right]_{d^{\prime} k^{\prime}} \Phi_{n M}^{k^{\prime} c}\left(\boldsymbol{r}^{\prime \prime \prime \prime \prime \prime \prime}, \boldsymbol{r}^{\prime \prime}\right) d \boldsymbol{r}^{\prime \prime \prime \prime \prime \prime} d \boldsymbol{r}^{\prime \prime \prime \prime \prime \prime \prime \prime} \\
& -\sum_{b^{\prime} c^{\prime} d^{\prime} k^{\prime}} \iiint \int \Phi_{M n}^{c b^{\prime}}\left(\boldsymbol{r}^{\prime \prime}, \boldsymbol{r}^{\prime \prime \prime}\right)\left[\tilde{\Phi}_{n n}^{-1}\left(\boldsymbol{r}^{\prime \prime \prime}, \boldsymbol{r}^{\prime \prime \prime \prime \prime}\right)\right]_{b^{\prime} c^{\prime}} \Phi_{M n}^{a d^{\prime}}\left(\boldsymbol{r}, \boldsymbol{r}^{\prime \prime \prime \prime \prime \prime}\right) \\
& \times\left[\tilde{\Phi}_{n n}^{-1}\left(\boldsymbol{r}^{\prime \prime \prime \prime \prime}, \boldsymbol{r}^{\prime \prime \prime \prime \prime \prime \prime}\right)\right]_{d^{\prime} k^{\prime}} \Phi_{n n}^{c^{\prime} k^{\prime}}\left(\boldsymbol{r}^{\prime \prime \prime \prime \prime \prime \prime \prime}, \boldsymbol{r}^{\prime \prime \prime \prime \prime}\right) d \boldsymbol{r}^{\prime \prime \prime \prime} d \boldsymbol{r}^{\prime \prime \prime \prime \prime} d \boldsymbol{r}^{\prime \prime \prime \prime \prime \prime} d \boldsymbol{r}^{\prime \prime \prime \prime \prime \prime \prime},
\end{aligned}
$$

where $\Phi_{M M}^{c a}\left(\boldsymbol{r}^{\prime \prime}, \boldsymbol{r}\right)$ is the equilibrium correlation function "density of magnetic moment - density of magnetic moment" of particles of sort $c, a, \Phi_{M n}^{c b^{\prime}}\left(\boldsymbol{r}^{\prime \prime}, \boldsymbol{r}^{\prime \prime \prime}\right)$ are the equilibrium correlation functions describing the magnetostrictive properties in the system. Functions $\left[\tilde{\Phi}_{\bar{g} \bar{g}}^{-1}\left(\boldsymbol{r}^{\prime}, \boldsymbol{r}^{\prime \prime}, \boldsymbol{r}^{\prime \prime \prime}, \boldsymbol{r}^{\prime \prime \prime \prime}\right)\right]_{b c c^{\prime} d^{\prime}}$, $\left[\tilde{\Phi}_{\bar{G} \bar{G}}^{-1}\left(\boldsymbol{r}^{\prime}, \boldsymbol{r}^{\prime \prime}, \boldsymbol{r}^{\prime \prime \prime}, \boldsymbol{r}^{\prime \prime \prime \prime}\right)\right]_{b c c^{\prime} d^{\prime}}$ are found from the corresponding integral relations

$$
\begin{aligned}
& \sum_{c^{\prime} d^{\prime}} \iint\left[\tilde{\Phi}_{\bar{g} \bar{g}}^{-1}\left(\boldsymbol{r}^{\prime}, \boldsymbol{r}^{\prime \prime}, \boldsymbol{r}^{\prime \prime \prime}, \boldsymbol{r}^{\prime \prime \prime \prime}\right)\right]_{b c c^{\prime} d^{\prime}} \Phi_{\bar{g} \bar{g}}^{c^{\prime} d^{\prime} a d}\left(\boldsymbol{r}^{\prime \prime \prime}, \boldsymbol{r}^{\prime \prime \prime \prime} ; \boldsymbol{r}, \boldsymbol{r}^{\prime \prime \prime \prime \prime \prime}\right) d \boldsymbol{r}^{\prime \prime \prime} d \boldsymbol{r}^{\prime \prime \prime \prime}=\delta_{b c a d} \delta\left(\boldsymbol{r}^{\prime}-\boldsymbol{r}\right) \delta\left(\boldsymbol{r}^{\prime \prime}-\boldsymbol{r}^{\prime \prime \prime \prime \prime \prime}\right), \\
& \sum_{c^{\prime} d^{\prime}} \iint\left[\tilde{\Phi}_{\bar{G} \bar{G}}^{-1}\left(\boldsymbol{r}^{\prime}, \boldsymbol{r}^{\prime \prime}, \boldsymbol{r}^{\prime \prime \prime}, \boldsymbol{r}^{\prime \prime \prime \prime \prime}\right)\right]_{b c c^{\prime} d^{\prime}} \Phi_{\bar{G} \bar{G}}^{c^{\prime} d^{\prime} a d}\left(\boldsymbol{r}^{\prime \prime \prime \prime}, \boldsymbol{r}^{\prime \prime \prime \prime \prime} ; \boldsymbol{r}, \boldsymbol{r}^{\prime \prime \prime \prime \prime \prime}\right) d \boldsymbol{r}^{\prime \prime \prime} d \boldsymbol{r}^{\prime \prime \prime \prime \prime}=\delta_{b c a d} \delta\left(\boldsymbol{r}^{\prime}-\boldsymbol{r}\right) \delta\left(\boldsymbol{r}^{\prime \prime}-\boldsymbol{r}^{\prime \prime \prime \prime \prime \prime}\right)
\end{aligned}
$$

under condition of calculating the fourth order correlation functions $\Phi_{\bar{g}}^{c^{\prime} d^{\prime} a d}\left(\boldsymbol{r}^{\prime \prime \prime}, \boldsymbol{r}^{\prime \prime \prime \prime} ; \boldsymbol{r}, \boldsymbol{r}^{\prime \prime \prime \prime \prime \prime}\right)$ $\Phi_{\bar{G}}^{c^{\prime} d^{\prime} a d}\left(\boldsymbol{r}^{\prime \prime \prime}, \boldsymbol{r}^{\prime \prime \prime \prime} ; \boldsymbol{r}, \boldsymbol{r}^{\prime \prime \prime \prime \prime \prime}\right)$ on variables of particle number density $\hat{n}_{a}(\boldsymbol{r})$ and magnetic moment density $\hat{\boldsymbol{M}}_{a}(\boldsymbol{r})$, which have the following structure:

$$
\begin{aligned}
& \Phi_{\bar{g} \bar{g}}^{c^{\prime} d^{\prime} a d}\left(\boldsymbol{r}^{\prime \prime \prime}, \boldsymbol{r}^{\prime \prime \prime \prime \prime} ; \boldsymbol{r}, \boldsymbol{r}^{\prime \prime \prime \prime \prime \prime}\right)=\left\langle\left(1-P_{n n}-P_{\bar{M} \bar{M}}\right) \hat{g}_{c^{\prime} d^{\prime}}\left(\boldsymbol{r}^{\prime \prime \prime}, \boldsymbol{r}^{\prime \prime \prime \prime \prime}\right)\left(1-P_{n n}-P_{\bar{M} \bar{M}}\right) \hat{g}_{a d}\left(\boldsymbol{r}, \boldsymbol{r}^{\prime \prime \prime \prime \prime \prime} ; \tau\right)\right\rangle_{0}, \\
& \Phi_{\bar{G} \bar{G}}^{c^{\prime} d^{\prime} a d}\left(\boldsymbol{r}^{\prime \prime \prime}, \boldsymbol{r}^{\prime \prime \prime \prime \prime} ; \boldsymbol{r}, \boldsymbol{r}^{\prime \prime \prime \prime \prime \prime}\right)=\left\langle\left(1-P_{n n}-P_{\bar{M} \bar{M}}-P_{\bar{g} \bar{g}} \hat{\leftrightarrow}_{\mathcal{G}^{\prime} d^{\prime}}\left(\boldsymbol{r}^{\prime \prime \prime}, \boldsymbol{r}^{\prime \prime \prime \prime}\right)\left(1-P_{n n}-P_{\bar{M} \bar{M}}-P_{\bar{g} \bar{g}}\right) \hat{\leftrightarrow}_{a d}\left(\boldsymbol{r}, \boldsymbol{r}^{\prime \prime \prime \prime \prime \prime} ; \tau\right)\right\rangle_{0} .\right.
\end{aligned}
$$


It is important to note that in this linear approximation the dynamic variables $\hat{n}_{a}(\boldsymbol{r}) \bar{M}_{a}(\boldsymbol{r})$, $\bar{g}_{a b}\left(\boldsymbol{r}, \boldsymbol{r}^{\prime}\right), \stackrel{\leftrightarrow}{G}_{a b}\left(\boldsymbol{r}, \boldsymbol{r}^{\prime}\right)$ are orthogonal in the sense of mean values:

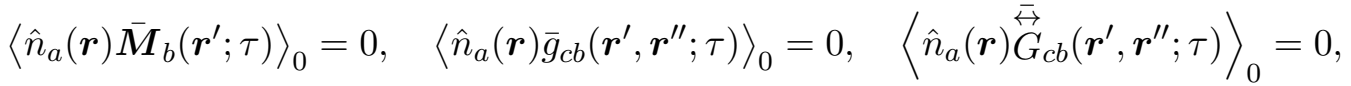

$$
\begin{aligned}
& \left\langle\overline{\boldsymbol{M}}_{a}(\boldsymbol{r}) \bar{g}_{c b}\left(\boldsymbol{r}^{\prime}, \boldsymbol{r}^{\prime \prime} ; \tau\right)\right\rangle_{0}=0, \quad\left\langle\overline{\boldsymbol{M}}_{a}(\boldsymbol{r}) \stackrel{\stackrel{\leftrightarrow}{G}}{c b}_{c b}\left(\boldsymbol{r}^{\prime}, \boldsymbol{r}^{\prime \prime} ; \tau\right)\right\rangle_{0}=0, \quad\left\langle\bar{g}_{a d}\left(\boldsymbol{r}^{\prime}, \boldsymbol{r}^{\prime \prime}\right) \stackrel{\leftrightarrow}{G}_{c b}\left(\boldsymbol{r}^{\prime \prime \prime}, \boldsymbol{r}^{\prime \prime \prime \prime} ; \tau\right)\right\rangle_{0}=0 .
\end{aligned}
$$

Since, expression for the relevant statistical operator is obtained in the linear approximation for fluctuations of the nonequilibrium thermodynamic parameters, we can construct a nonequilibrium statistical operator in this approximation. Substituting (19) into (7) after simple transformations, we obtain:

$$
\begin{aligned}
& \rho(t)=\rho_{q}^{0}(t)-\sum_{a b} \int d \boldsymbol{r}^{\prime} \int d \boldsymbol{r}^{\prime \prime} \int_{-\infty}^{t} e^{\varepsilon\left(t^{\prime}-t\right)} \hat{T}_{0}\left(t, t^{\prime}\right) \int_{0}^{1} d \tau\left(\rho_{0}^{\tau} I_{n}^{a}\left(\boldsymbol{r}^{\prime} ; t^{\prime}\right) \rho_{0}^{1-\tau}\right)\left[\tilde{\Phi}_{n n}^{-1}\left(\boldsymbol{r}^{\prime}, \boldsymbol{r}^{\prime \prime}\right)\right]_{a b}\left\langle\delta \hat{n}_{b}\left(\boldsymbol{r}^{\prime \prime}\right)\right\rangle^{t^{\prime}} d t^{\prime} \\
& -\sum_{a b} \int d \boldsymbol{r}^{\prime} \int d \boldsymbol{r}^{\prime \prime} \int_{-\infty}^{t} e^{\varepsilon\left(t^{\prime}-t\right)} \hat{T}_{0}\left(t, t^{\prime}\right) \int_{0}^{1} d \tau\left(\rho_{0}^{\tau} I_{M}^{a}\left(\boldsymbol{r}^{\prime} ; t^{\prime}\right) \rho_{0}^{1-\tau}\right)\left[\tilde{\Phi}_{\bar{M} \bar{M}}^{-1}\left(\boldsymbol{r}^{\prime}, \boldsymbol{r}^{\prime \prime}\right)\right]_{a b}\left\langle\delta \overline{\boldsymbol{M}}_{b}\left(\boldsymbol{r}^{\prime \prime}\right)\right\rangle^{t^{\prime}} d t^{\prime} \\
& -\sum_{a b c d} \int d \boldsymbol{r} \int d \boldsymbol{r}^{\prime} \int d \boldsymbol{r}^{\prime \prime} \int d \boldsymbol{r}^{\prime \prime \prime} \int_{-\infty}^{t} e^{\varepsilon\left(t^{\prime}-t\right)} \hat{T}_{0}\left(t, t^{\prime}\right) \int_{0}^{1} d \tau\left(\rho_{0}^{\tau} I_{G}^{a b}\left(\boldsymbol{r}, \boldsymbol{r}^{\prime} ; t^{\prime}\right) \rho_{0}^{1-\tau}\right) \\
& \times\left[\tilde{\Phi}_{\bar{G} \bar{G}}^{-1}\left(\boldsymbol{r}, \boldsymbol{r}^{\prime}, \boldsymbol{r}^{\prime \prime}, \boldsymbol{r}^{\prime \prime \prime}\right)\right]_{a b c d}\left\langle\delta \stackrel{\leftrightarrow}{G}_{c d}\left(\boldsymbol{r}^{\prime \prime}, \boldsymbol{r}^{\prime \prime \prime}\right)\right\rangle^{t^{\prime}} d t^{\prime} \\
& -\sum_{a b c d} \int d \boldsymbol{r} \int d \boldsymbol{r}^{\prime} \int d \boldsymbol{r}^{\prime \prime} \int d \boldsymbol{r}^{\prime \prime \prime} \int_{-\infty}^{t} e^{\varepsilon\left(t^{\prime}-t\right)} \hat{T}_{0}\left(t, t^{\prime}\right) \int_{0}^{1} d \tau\left(\rho_{0}^{\tau} I_{g}^{a b}\left(\boldsymbol{r}, \boldsymbol{r}^{\prime} ; t^{\prime}\right) \rho_{0}^{1-\tau}\right) \\
& \times\left[\tilde{\Phi}_{\bar{g} \bar{g}}^{-1}\left(\boldsymbol{r}, \boldsymbol{r}^{\prime}, \boldsymbol{r}^{\prime \prime}, \boldsymbol{r}^{\prime \prime \prime}\right)\right]_{a b c d}\left\langle\delta \bar{g}_{c d}\left(\boldsymbol{r}^{\prime \prime}, \boldsymbol{r}^{\prime \prime \prime}\right)\right\rangle^{t^{\prime}} d t^{\prime},
\end{aligned}
$$

where

$$
\begin{gathered}
I_{n}^{a}\left(\boldsymbol{r}^{\prime} t^{\prime}\right)=(1-P) i \hat{L}\left(t^{\prime}\right) \hat{n}_{a}\left(\boldsymbol{r}^{\prime}\right), \quad I_{M}^{a}\left(\boldsymbol{r}^{\prime} ; t^{\prime}\right)=(1-P) i \hat{L}\left(t^{\prime}\right) \hat{\boldsymbol{M}}_{a}\left(\boldsymbol{r}^{\prime}\right), \\
I_{G}^{a b}\left(\boldsymbol{r}, \boldsymbol{r}^{\prime} ; t^{\prime}\right)=(1-P) i \hat{L}\left(t^{\prime}\right) \stackrel{\leftrightarrow}{G}_{a b}\left(\boldsymbol{r}, \boldsymbol{r}^{\prime}\right), \quad I_{g}^{a b}\left(\boldsymbol{r}, \boldsymbol{r}^{\prime} ; t^{\prime}\right)=(1-P) i \hat{L}\left(t^{\prime}\right) \hat{g}_{a b}\left(\boldsymbol{r}, \boldsymbol{r}^{\prime}\right)
\end{gathered}
$$

are the microscopic generalized fluxes describing diffusion, magneto-diffusion and reaction weakly nonequilibrium processes in the system and on which the generalized transfer coefficients and functions of reactions between components are built. $P$ is the generalized Mori projection operator in the linear approximation by fluctuations and has the following structure:

$$
\begin{aligned}
P(\ldots)= & \sum_{a b} \int d \boldsymbol{r}^{\prime} \int d \boldsymbol{r}^{\prime \prime}\left\langle\ldots \hat{n}_{a}\left(\boldsymbol{r}^{\prime} ; \tau\right)\right\rangle_{0}\left[\tilde{\Phi}_{n n}^{-1}\left(\boldsymbol{r}^{\prime}, \boldsymbol{r}^{\prime \prime}\right)\right]_{a b} \hat{n}_{b}\left(\boldsymbol{r}^{\prime \prime}\right) \\
& +\sum_{a b} \int d \boldsymbol{r}^{\prime} \int d \boldsymbol{r}^{\prime \prime}\left\langle\ldots \overline{\boldsymbol{M}}_{a}\left(\boldsymbol{r}^{\prime} ; \tau\right)\right\rangle_{0}\left[\tilde{\Phi}_{\bar{M}}^{-1} \bar{M}^{(}\left(\boldsymbol{r}^{\prime}, \boldsymbol{r}^{\prime \prime}\right)\right]_{a b} \overline{\boldsymbol{M}}_{b}\left(\boldsymbol{r}^{\prime \prime}\right) \\
& +\sum_{a b c d} \int d \boldsymbol{r} \int d \boldsymbol{r}^{\prime} \int d \boldsymbol{r}^{\prime \prime} \int d \boldsymbol{r}^{\prime \prime \prime}\left\langle\ldots \stackrel{\bar{G}}{G}_{a b}\left(\boldsymbol{r}, \boldsymbol{r}^{\prime} ; \tau\right)\right\rangle_{0}\left[\tilde{\Phi}_{\bar{G} \bar{G}}^{-1}\left(\boldsymbol{r}, \boldsymbol{r}^{\prime}, \boldsymbol{r}^{\prime \prime}, \boldsymbol{r}^{\prime \prime \prime}\right)\right]_{a b c d} \stackrel{\bar{G}}{G d}_{c d}\left(\boldsymbol{r}^{\prime \prime}, \boldsymbol{r}^{\prime \prime \prime}\right) \\
& +\sum_{a b c d} \int d \boldsymbol{r} \int d \boldsymbol{r}^{\prime} \int d \boldsymbol{r}^{\prime \prime} \int d \boldsymbol{r}^{\prime \prime \prime}\left\langle\ldots \bar{g}_{a b}\left(\boldsymbol{r}, \boldsymbol{r}^{\prime} ; \tau\right)\right\rangle_{0}\left[\tilde{\Phi}_{\bar{g} \bar{g}}^{-1}\left(\boldsymbol{r}, \boldsymbol{r}^{\prime}, \boldsymbol{r}^{\prime \prime}, \boldsymbol{r}^{\prime \prime \prime}\right)\right]_{a b c d} \bar{g}_{c d}\left(\boldsymbol{r}^{\prime \prime}, \boldsymbol{r}^{\prime \prime \prime}\right) .
\end{aligned}
$$

$\hat{T}_{0}\left(t, t^{\prime}\right)$ is the evolution operator taking into account the design procedure in the linear approximation by fluctuations. The time dependence of the generalized fluxes (33) is related to the action of the Liouville operator $i \hat{L}\left(t^{\prime}\right)$, which corresponds to the Hamiltonian of the system (5) that contains an external time-dependent magnetic field. By structure, the nonequilibrium statistical operator (32) is a linear functional by fluctuations of the abbreviated description parameters $\left\langle\delta \hat{n}_{b}\left(\boldsymbol{r}^{\prime \prime}\right)\right\rangle^{t^{\prime}},\left\langle\delta \overline{\boldsymbol{M}}_{b}\left(\boldsymbol{r}^{\prime \prime}\right)\right\rangle^{t^{\prime}}$, 


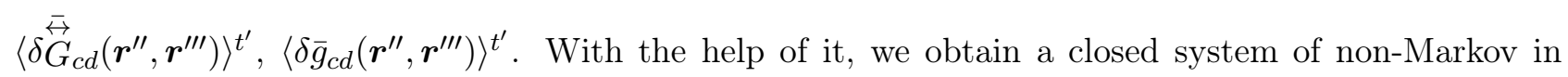
time integro-differential equations for fluctuations of the abbreviated description parameters:

$$
\begin{aligned}
& \frac{\partial}{\partial t}\left\langle\delta \hat{n}_{a}(\boldsymbol{r})\right\rangle^{t}=-\sum_{b} \int d \boldsymbol{r}^{\prime} \int d \boldsymbol{r}^{\prime \prime} \int_{-\infty}^{t} e^{\varepsilon\left(t^{\prime}-t\right)} \frac{\partial}{\partial \boldsymbol{r}} \bar{D}_{n n}^{a b}\left(\boldsymbol{r}, \boldsymbol{r}^{\prime} ; t, t^{\prime}\right) \frac{\partial}{\partial \boldsymbol{r}^{\prime}}\left[\tilde{\Phi}_{n n}^{-1}\left(\boldsymbol{r}^{\prime}, \boldsymbol{r}^{\prime \prime}\right)\right]_{b c}\left\langle\delta \hat{n}_{c}\left(\boldsymbol{r}^{\prime \prime}\right)\right\rangle^{t^{\prime}} d t^{\prime} \\
& +\sum_{b c} \int d \boldsymbol{r}^{\prime} \int d \boldsymbol{r}^{\prime \prime} \int_{-\infty}^{t} e^{\varepsilon\left(t^{\prime}-t\right)} \frac{\partial}{\partial \boldsymbol{r}} \bar{D}_{n R}^{a b}\left(\boldsymbol{r}, \boldsymbol{r}^{\prime} ; t, t^{\prime}\right) \frac{\partial}{\partial \boldsymbol{r}^{\prime}}\left[\tilde{\Phi}_{\bar{M} \bar{M}}^{-1}\left(\boldsymbol{r}^{\prime}, \boldsymbol{r}^{\prime \prime}\right)\right]_{b c}\left\langle\delta \overline{\boldsymbol{M}}_{c}\left(\boldsymbol{r}^{\prime \prime}\right)\right\rangle^{t^{\prime}} d t^{\prime} \\
& +\sum_{b c} \int d \boldsymbol{r}^{\prime} \int d \boldsymbol{r}^{\prime \prime} \int_{-\infty}^{t} e^{\varepsilon\left(t^{\prime}-t\right)} \frac{\partial}{\partial \boldsymbol{r}} \bar{D}_{n M}^{a b}\left(\boldsymbol{r}, \boldsymbol{r}^{\prime} ; t, t^{\prime}\right)\left(\left[\tilde{\Phi}_{\bar{M} \bar{M}}^{-1}\left(\boldsymbol{r}^{\prime}, \boldsymbol{r}^{\prime \prime}\right)\right]_{b c}\left\langle\delta \overline{\boldsymbol{M}}_{c}\left(\boldsymbol{r}^{\prime \prime}\right)\right\rangle^{t^{\prime}}+\beta \boldsymbol{B}\left(\boldsymbol{r}^{\prime}, t^{\prime}\right)\right) d t^{\prime} \\
& -\sum_{b c} \int d \boldsymbol{r}^{\prime} \int d \boldsymbol{r}^{\prime \prime} \int d \boldsymbol{r}^{\prime \prime \prime} \int d \boldsymbol{r}^{\prime \prime \prime \prime} \int_{-\infty}^{t} e^{\varepsilon\left(t^{\prime}-t\right)} \frac{\partial}{\partial \boldsymbol{r}} \bar{K}_{n G}^{a b c}\left(\boldsymbol{r}, \boldsymbol{r}^{\prime}, \boldsymbol{r}^{\prime \prime} ; t, t^{\prime}\right) \\
& \times\left[\tilde{\Phi}_{\bar{G} \bar{G}}^{-1}\left(\boldsymbol{r}^{\prime}, \boldsymbol{r}^{\prime \prime}, \boldsymbol{r}^{\prime \prime \prime}, \boldsymbol{r}^{\prime \prime \prime \prime}\right)\right]_{b c d c^{\prime}}\left\langle\dot{G}_{d c^{\prime}}\left(\boldsymbol{r}^{\prime \prime \prime}, \boldsymbol{r}^{\prime \prime \prime \prime}\right)\right\rangle^{t^{\prime}} d t^{\prime} \\
& -\sum_{b c} \int d \boldsymbol{r}^{\prime} \int d \boldsymbol{r}^{\prime \prime} \int d \boldsymbol{r}^{\prime \prime \prime} \int d \boldsymbol{r}^{\prime \prime \prime \prime} \int_{-\infty}^{t} e^{\varepsilon\left(t^{\prime}-t\right)} \frac{\partial}{\partial \boldsymbol{r}} \bar{K}_{n g}^{a b c}\left(\boldsymbol{r}, \boldsymbol{r}^{\prime}, \boldsymbol{r}^{\prime \prime} ; t, t^{\prime}\right) \\
& \times\left[\tilde{\Phi}_{\bar{g} \bar{g}}^{-1}\left(\boldsymbol{r}^{\prime}, \boldsymbol{r}^{\prime \prime}, \boldsymbol{r}^{\prime \prime \prime}, \boldsymbol{r}^{\prime \prime \prime \prime}\right)\right]_{b c d c^{\prime}}\left\langle\delta \bar{g}_{d c^{\prime}}\left(\boldsymbol{r}^{\prime \prime \prime}, \boldsymbol{r}^{\prime \prime \prime \prime}\right)\right\rangle^{t^{\prime}} d t^{\prime}, \\
& \frac{\partial}{\partial t}\left\langle\delta \hat{\boldsymbol{M}}_{a}(\boldsymbol{r})\right\rangle^{t}=-\sum_{b c} \int d \boldsymbol{r}^{\prime} \int d \boldsymbol{r}^{\prime \prime}\left\langle\frac{i}{h}\left[\hat{\boldsymbol{M}}_{a}(\boldsymbol{r}), \hat{\boldsymbol{M}}_{b}\left(\boldsymbol{r}^{\prime}\right)\right]\right\rangle_{0}^{t}\left(\beta^{-1}\left[\tilde{\Phi}_{\bar{M} \bar{M}}^{-1}\left(\boldsymbol{r}^{\prime}, \boldsymbol{r}^{\prime \prime}\right)\right]_{b c}\left\langle\delta \overline{\boldsymbol{M}}_{c}\left(\boldsymbol{r}^{\prime \prime}\right)\right\rangle^{t}+\boldsymbol{B}\left(\boldsymbol{r}^{\prime}, t\right)\right) \\
& +\sum_{b c} \int d \boldsymbol{r}^{\prime} \int d \boldsymbol{r}^{\prime \prime} \int_{-\infty}^{t} e^{\varepsilon\left(t^{\prime}-t\right)} \bar{D}_{M n}^{a b}\left(\boldsymbol{r}, \boldsymbol{r}^{\prime} ; t, t^{\prime}\right) \frac{\partial}{\partial \boldsymbol{r}^{\prime}}\left[\tilde{\Phi}_{n n}^{-1}\left(\boldsymbol{r}^{\prime}, \boldsymbol{r}^{\prime \prime}\right)\right]_{b c}\left\langle\delta \hat{n}_{c}\left(\boldsymbol{r}^{\prime \prime}\right)\right\rangle^{t^{\prime}} d t^{\prime} \\
& +\sum_{b c} \int d \boldsymbol{r}^{\prime} \int d \boldsymbol{r}^{\prime \prime} \int_{-\infty}^{t} e^{\varepsilon\left(t^{\prime}-t\right)} \bar{D}_{M M}^{a b}\left(\boldsymbol{r}, \boldsymbol{r}^{\prime} ; t, t^{\prime}\right)\left(\left[\tilde{\Phi}_{\bar{M} \bar{M}}^{-1}\left(\boldsymbol{r}^{\prime}, \boldsymbol{r}^{\prime \prime}\right)\right]_{b c}\left\langle\delta \overline{\boldsymbol{M}}_{c}\left(\boldsymbol{r}^{\prime \prime}\right)\right\rangle^{t^{\prime}}+\beta \boldsymbol{B}\left(\boldsymbol{r}^{\prime}, t^{\prime}\right)\right) d t^{\prime} \\
& +\sum_{b c d c^{\prime}} \int d \boldsymbol{r}^{\prime} \int d \boldsymbol{r}^{\prime \prime} \int d \boldsymbol{r}^{\prime \prime \prime} \int d \boldsymbol{r}^{\prime \prime \prime \prime} \int_{-\infty}^{t} e^{\varepsilon\left(t^{\prime}-t\right)} \bar{K}_{M G}^{a b c}\left(\boldsymbol{r}, \boldsymbol{r}^{\prime}, \boldsymbol{r}^{\prime \prime} ; t, t^{\prime}\right)
\end{aligned}
$$

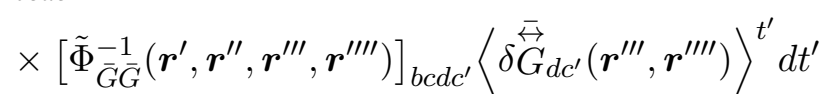

$$
\begin{aligned}
& +\sum_{b c d c^{\prime}} \int d \boldsymbol{r}^{\prime} \int d \boldsymbol{r}^{\prime \prime} \int d \boldsymbol{r}^{\prime \prime \prime} \int d \boldsymbol{r}^{\prime \prime \prime \prime} \int_{-\infty}^{t} e^{\varepsilon\left(t^{\prime}-t\right)} \bar{K}_{M g}^{a b c}\left(\boldsymbol{r}, \boldsymbol{r}^{\prime}, \boldsymbol{r}^{\prime \prime} ; t, t^{\prime}\right) \\
& \times\left[\tilde{\Phi}_{\bar{g} \bar{g}}^{-1}\left(\boldsymbol{r}^{\prime}, \boldsymbol{r}^{\prime \prime}, \boldsymbol{r}^{\prime \prime \prime}, \boldsymbol{r}^{\prime \prime \prime \prime}\right)\right]_{b c d c^{\prime}}\left\langle\delta \bar{g}_{d c^{\prime}}\left(\boldsymbol{r}^{\prime \prime \prime}, \boldsymbol{r}^{\prime \prime \prime \prime}\right)\right\rangle^{t^{\prime}} d t^{\prime}, \\
& \frac{\partial}{\partial t}\left\langle\delta \stackrel{\bar{G}}{G}_{a b}\left(\boldsymbol{r}, \boldsymbol{r}^{\prime}\right)\right\rangle^{t}=\left\langle i \hat{L}\left(t^{\prime}\right) \delta \stackrel{\overleftarrow{G}}{G}_{a b}\left(\boldsymbol{r}, \boldsymbol{r}^{\prime}\right)\right\rangle_{q 0}^{t} \\
& -\sum_{c d} \int d \boldsymbol{r}^{\prime \prime} \int d \boldsymbol{r}^{\prime \prime \prime} \int_{-\infty}^{t} e^{\varepsilon\left(t^{\prime}-t\right)} \bar{K}_{G n}^{a b c}\left(\boldsymbol{r}, \boldsymbol{r}^{\prime}, \boldsymbol{r}^{\prime \prime} ; t, t^{\prime}\right) \frac{\partial}{\partial \boldsymbol{r}^{\prime \prime}}\left[\tilde{\Phi}_{n n}^{-1}\left(\boldsymbol{r}^{\prime \prime}, \boldsymbol{r}^{\prime \prime \prime}\right)\right]_{c d}\left\langle\delta \hat{n}_{d}\left(\boldsymbol{r}^{\prime \prime \prime}\right)\right\rangle^{t^{\prime}} d t^{\prime} \\
& +\sum_{c d} \int d \boldsymbol{r}^{\prime \prime} \int d \boldsymbol{r}^{\prime \prime \prime} \int_{-\infty}^{t} e^{\varepsilon\left(t^{\prime}-t\right)} \bar{K}_{G R}^{a b c}\left(\boldsymbol{r}, \boldsymbol{r}^{\prime}, \boldsymbol{r}^{\prime \prime} ; t, t^{\prime}\right) \frac{\partial}{\partial \boldsymbol{r}^{\prime \prime}}\left[\tilde{\Phi}_{\bar{M} \bar{M}}^{-1}\left(\boldsymbol{r}^{\prime \prime}, \boldsymbol{r}^{\prime \prime \prime}\right)\right]_{c d}\left\langle\delta \overline{\boldsymbol{M}}_{d}\left(\boldsymbol{r}^{\prime \prime \prime}\right)\right\rangle^{t^{\prime}} d t^{\prime} \\
& +\sum_{c d} \int d \boldsymbol{r}^{\prime \prime} \int d \boldsymbol{r}^{\prime \prime \prime} \int_{-\infty}^{t} e^{\varepsilon\left(t^{\prime}-t\right)} \bar{K}_{G M}^{a b c}\left(\boldsymbol{r}, \boldsymbol{r}^{\prime}, \boldsymbol{r}^{\prime \prime} ; t, t^{\prime}\right)\left(\left[\tilde{\Phi}_{\bar{M} \bar{M}}^{-1}\left(\boldsymbol{r}^{\prime \prime}, \boldsymbol{r}^{\prime \prime \prime}\right)\right]_{c d}\left\langle\delta \overline{\boldsymbol{M}}_{d}\left(\boldsymbol{r}^{\prime \prime \prime}\right)\right\rangle^{t^{\prime}}+\beta \boldsymbol{B}\left(\boldsymbol{r}^{\prime}, t^{\prime}\right)\right) d t^{\prime} \\
& +\sum_{d c c^{\prime} d^{\prime}} \int d \boldsymbol{r}^{\prime \prime} \int d \boldsymbol{r}^{\prime \prime \prime} \int d \boldsymbol{r}^{\prime \prime \prime \prime} \int d \boldsymbol{r}^{\prime \prime \prime \prime \prime \prime} \int_{-\infty}^{t} e^{\varepsilon\left(t^{\prime}-t\right)} \bar{K}_{G G}^{a b c d}\left(\boldsymbol{r}, \boldsymbol{r}^{\prime}, \boldsymbol{r}^{\prime \prime}, \boldsymbol{r}^{\prime \prime \prime} ; t, t^{\prime}\right)
\end{aligned}
$$

Mathematical Modeling and Computing, Vol. 8, No. 2, pp. 267-281 (2021) 


$$
\begin{aligned}
& \times\left[\tilde{\Phi}_{\bar{G} \bar{G}}^{-1}\left(\boldsymbol{r}^{\prime \prime}, \boldsymbol{r}^{\prime \prime \prime}, \boldsymbol{r}^{\prime \prime \prime \prime \prime}, \boldsymbol{r}^{\prime \prime \prime \prime \prime \prime}\right)\right]_{c d c^{\prime} d^{\prime}}\left\langle\delta \stackrel{\leftrightarrow}{G}_{c^{\prime} d^{\prime}}\left(\boldsymbol{r}^{\prime \prime \prime \prime \prime}, \boldsymbol{r}^{\prime \prime \prime \prime \prime \prime}\right)\right\rangle^{t^{\prime}} d t^{\prime} \\
& +\sum_{d c c^{\prime} d^{\prime}} \int d \boldsymbol{r}^{\prime \prime} \int d \boldsymbol{r}^{\prime \prime \prime} \int d \boldsymbol{r}^{\prime \prime \prime \prime} \int d \boldsymbol{r}^{\prime \prime \prime \prime \prime} \int_{-\infty}^{t} e^{\varepsilon\left(t^{\prime}-t\right)} \bar{K}_{G g}^{a b c d}\left(\boldsymbol{r}, \boldsymbol{r}^{\prime}, \boldsymbol{r}^{\prime \prime} \boldsymbol{r}^{\prime \prime \prime} ; t, t^{\prime}\right) \\
& \times\left[\tilde{\Phi}_{\bar{g} \bar{g}}^{-1}\left(\boldsymbol{r}^{\prime \prime}, \boldsymbol{r}^{\prime \prime \prime}, \boldsymbol{r}^{\prime \prime \prime \prime \prime}, \boldsymbol{r}^{\prime \prime \prime \prime \prime \prime}\right)\right]_{c d c^{\prime} d^{\prime}}\left\langle\delta \bar{g}_{c^{\prime} d^{\prime}}\left(\boldsymbol{r}^{\prime \prime \prime \prime \prime}, \boldsymbol{r}^{\prime \prime \prime \prime \prime \prime}\right)\right\rangle^{t^{\prime}} d t^{\prime}, \\
& \frac{\partial}{\partial t}\left\langle\delta \bar{g}_{a b}\left(\boldsymbol{r}, \boldsymbol{r}^{\prime}\right)\right\rangle^{t}=\left\langle i \hat{L}\left(t^{\prime}\right) \delta \bar{g}_{a b}\left(\boldsymbol{r}, \boldsymbol{r}^{\prime}\right)\right\rangle_{q 0}^{t} \\
& -\sum_{c d} \int d \boldsymbol{r}^{\prime \prime} \int d \boldsymbol{r}^{\prime \prime \prime} \int_{-\infty}^{t} e^{\varepsilon\left(t^{\prime}-t\right)} \bar{K}_{g n}^{a b c}\left(\boldsymbol{r}, \boldsymbol{r}^{\prime}, \boldsymbol{r}^{\prime \prime} ; t, t^{\prime}\right) \frac{\partial}{\partial \boldsymbol{r}^{\prime \prime}}\left[\tilde{\Phi}_{n n}^{-1}\left(\boldsymbol{r}^{\prime \prime}, \boldsymbol{r}^{\prime \prime \prime}\right)\right]_{c d}\left\langle\delta \hat{n}_{d}\left(\boldsymbol{r}^{\prime \prime \prime}\right)\right\rangle^{t^{\prime}} d t^{\prime} \\
& +\sum_{c d} \int d \boldsymbol{r}^{\prime \prime} \int d \boldsymbol{r}^{\prime \prime \prime} \int_{-\infty}^{t} e^{\varepsilon\left(t^{\prime}-t\right)} \bar{K}_{g R}^{a b c}\left(\boldsymbol{r}, \boldsymbol{r}^{\prime}, \boldsymbol{r}^{\prime \prime} ; t, t^{\prime}\right) \frac{\partial}{\partial \boldsymbol{r}^{\prime \prime}}\left[\tilde{\Phi}_{\bar{M} \bar{M}}^{-1}\left(\boldsymbol{r}^{\prime \prime}, \boldsymbol{r}^{\prime \prime \prime}\right)\right]_{c d}\left\langle\delta \overline{\boldsymbol{M}}_{d}\left(\boldsymbol{r}^{\prime \prime \prime}\right)\right\rangle^{t^{\prime}} d t^{\prime} \\
& +\sum_{c d} \int d \boldsymbol{r}^{\prime \prime} \int d \boldsymbol{r}^{\prime \prime \prime} \int_{-\infty}^{t} e^{\varepsilon\left(t^{\prime}-t\right)} \bar{K}_{g M}^{a b c}\left(\boldsymbol{r}, \boldsymbol{r}^{\prime}, \boldsymbol{r}^{\prime \prime} ; t, t^{\prime}\right)\left(\left[\tilde{\Phi}_{\bar{M} \bar{M}}^{-1}\left(\boldsymbol{r}^{\prime \prime}, \boldsymbol{r}^{\prime \prime \prime}\right)\right]_{c d}\left\langle\delta \overline{\boldsymbol{M}}_{d}\left(\boldsymbol{r}^{\prime \prime \prime}\right)\right\rangle^{t^{\prime}}+\beta \boldsymbol{B}\left(\boldsymbol{r}^{\prime}, t^{\prime}\right)\right) d t^{\prime} \\
& +\sum_{d c c^{\prime} d^{\prime}} \int d \boldsymbol{r}^{\prime \prime} \int d \boldsymbol{r}^{\prime \prime \prime} \int d \boldsymbol{r}^{\prime \prime \prime \prime} \int d \boldsymbol{r}^{\prime \prime \prime \prime \prime} \int_{-\infty}^{t} e^{\varepsilon\left(t^{\prime}-t\right)} \bar{K}_{g G}^{a b c d}\left(\boldsymbol{r}, \boldsymbol{r}^{\prime}, \boldsymbol{r}^{\prime \prime}, \boldsymbol{r}^{\prime \prime \prime} ; t, t^{\prime}\right)
\end{aligned}
$$

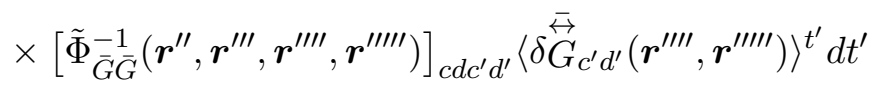

$$
\begin{aligned}
& +\sum_{d c c^{\prime} d^{\prime}} \int d \boldsymbol{r}^{\prime \prime} \int d \boldsymbol{r}^{\prime \prime \prime} \int d \boldsymbol{r}^{\prime \prime \prime \prime} \int d \boldsymbol{r}^{\prime \prime \prime \prime \prime} \int_{-\infty}^{t} e^{\varepsilon\left(t^{\prime}-t\right)} \bar{K}_{g g}^{a b c d}\left(\boldsymbol{r}, \boldsymbol{r}^{\prime}, \boldsymbol{r}^{\prime \prime} \boldsymbol{r}^{\prime \prime \prime} ; t, t^{\prime}\right) \\
& \times\left[\tilde{\Phi}_{\bar{g} \bar{g}}^{-1}\left(\boldsymbol{r}^{\prime \prime}, \boldsymbol{r}^{\prime \prime \prime}, \boldsymbol{r}^{\prime \prime \prime \prime}, \boldsymbol{r}^{\prime \prime \prime \prime \prime \prime}\right)\right]_{c d c^{\prime} d^{\prime}}\left\langle\delta \bar{g}_{c^{\prime} d^{\prime}}\left(\boldsymbol{r}^{\prime \prime \prime \prime}, \boldsymbol{r}^{\prime \prime \prime \prime \prime \prime}\right)\right\rangle^{t^{\prime}} d t^{\prime},
\end{aligned}
$$

where $\langle\ldots\rangle_{q 0}^{t}=\operatorname{Sp}\left(\ldots \rho_{q}^{0}(t)\right), \bar{D}_{l j}$ are the transfer kernels and functions of reactions $\bar{K}_{l j k}, \bar{K}_{l j k s}$, in which the averaging operation is performed with an equilibrium statistical operator (19), $\frac{\partial}{\partial \boldsymbol{r}} \equiv \operatorname{grad}_{\boldsymbol{r}}$. The obtained system of transfer equations is closed and contains the non-dissipative and dissipative contributions, which describe non-Markov diffusion, magneto-diffusion and reaction catalytic processes. If magnetic processes are not formally taken into account, we obtain a system of equations for description of reaction-diffusion processes that were obtained in one form or another [42-44]:

$$
\begin{aligned}
\frac{\partial}{\partial t}\left\langle\delta \hat{n}_{a}(\boldsymbol{r})\right\rangle^{t}= & -\sum_{b} \int d \boldsymbol{r}^{\prime} \int d \boldsymbol{r}^{\prime \prime} \int_{-\infty}^{t} e^{\varepsilon\left(t^{\prime}-t\right)} \frac{\partial}{\partial \boldsymbol{r}} \bar{D}_{n n}^{a b}\left(\boldsymbol{r}, \boldsymbol{r}^{\prime} ; t, t^{\prime}\right) \frac{\partial}{\partial \boldsymbol{r}^{\prime}}\left[\tilde{\Phi}_{n n}^{-1}\left(\boldsymbol{r}^{\prime}, \boldsymbol{r}^{\prime \prime}\right)\right]_{b c}\left\langle\delta \hat{n}_{c}\left(\boldsymbol{r}^{\prime \prime}\right)\right\rangle^{t^{\prime}} d t^{\prime} \\
& -\sum_{b c} \int d \boldsymbol{r}^{\prime} \int d \boldsymbol{r}^{\prime \prime} \int d \boldsymbol{r}^{\prime \prime \prime} \int d \boldsymbol{r}^{\prime \prime \prime \prime \prime} \int_{-\infty}^{t} e^{\varepsilon\left(t^{\prime}-t\right)} \frac{\partial}{\partial \boldsymbol{r}} \bar{K}_{n g}^{a b c}\left(\boldsymbol{r}, \boldsymbol{r}^{\prime}, \boldsymbol{r}^{\prime \prime} ; t, t^{\prime}\right) \\
& \times\left[\tilde{\Phi}_{\bar{g} \bar{g}}^{-1}\left(\boldsymbol{r}^{\prime}, \boldsymbol{r}^{\prime \prime}, \boldsymbol{r}^{\prime \prime \prime}, \boldsymbol{r}^{\prime \prime \prime \prime}\right)\right]_{b c d c^{\prime}}\left\langle\delta \bar{g}_{d c^{\prime}}\left(\boldsymbol{r}^{\prime \prime \prime}, \boldsymbol{r}^{\prime \prime \prime \prime}\right)\right\rangle^{t^{\prime}} d t^{\prime}, \\
\frac{\partial}{\partial t}\left\langle\delta \bar{g}_{a b}\left(\boldsymbol{r}, \boldsymbol{r}^{\prime}\right)\right\rangle^{t}= & \left\langle i \hat{L}\left(t^{\prime}\right) \delta \bar{g}_{a b}\left(\boldsymbol{r}, \boldsymbol{r}^{\prime}\right)\right\rangle_{q 0}^{t} \\
& -\sum_{c d} \int d \boldsymbol{r}^{\prime \prime} \int d \boldsymbol{r}^{\prime \prime \prime} \int_{-\infty}^{t} e^{\varepsilon\left(t^{\prime}-t\right)} \bar{K}_{g n}^{a b c}\left(\boldsymbol{r}, \boldsymbol{r}^{\prime}, \boldsymbol{r}^{\prime \prime} ; t, t^{\prime}\right) \frac{\partial}{\partial \boldsymbol{r}^{\prime \prime}}\left[\tilde{\Phi}_{n n}^{-1}\left(\boldsymbol{r}^{\prime \prime}, \boldsymbol{r}^{\prime \prime \prime}\right)\right]_{c d}\left\langle\delta \hat{n}_{d}\left(\boldsymbol{r}^{\prime \prime \prime}\right)\right\rangle^{t^{\prime}} d t^{\prime} \\
& +\sum_{d c c^{\prime} d^{\prime}} \int d \boldsymbol{r}^{\prime \prime} \int d \boldsymbol{r}^{\prime \prime \prime \prime} \int d \boldsymbol{r}^{\prime \prime \prime \prime \prime} \int d \boldsymbol{r}^{\prime \prime \prime \prime \prime \prime} \int_{-\infty}^{t} e^{\varepsilon\left(t^{\prime}-t\right)} \bar{K}_{g g}^{a b c d}\left(\boldsymbol{r}, \boldsymbol{r}^{\prime}, \boldsymbol{r}^{\prime \prime} \boldsymbol{r}^{\prime \prime \prime \prime} ; t, t^{\prime}\right) \\
& \times\left[\tilde{\Phi}_{\bar{g} \bar{g}}^{-1}\left(\boldsymbol{r}^{\prime \prime}, \boldsymbol{r}^{\prime \prime \prime}, \boldsymbol{r}^{\prime \prime \prime \prime \prime}, \boldsymbol{r}^{\prime \prime \prime \prime \prime \prime}\right)\right]_{c d c^{\prime} d^{\prime}}\left\langle\delta \bar{g}_{c^{\prime} d^{\prime}}\left(\boldsymbol{r}^{\prime \prime \prime \prime \prime}, \boldsymbol{r}^{\prime \prime \prime \prime \prime \prime}\right)\right\rangle^{t^{\prime}} d t^{\prime} .
\end{aligned}
$$




\section{Conclusions}

In this paper a statistical description of catalytic oxidation processes is proposed taking into account the reaction-diffusion processes for magnetoactive ions (anions), atoms adsorbed on the metal surface taking into account electronic subsystem. The quantum-classical model for description of reactiondiffusion processes for magnetoactive ions and atoms adsorbed on the metal surface is considered for the purpose of its further application to studies of hydrogen oxidation processes on the surface of magnetoactive metals. As a result, the Hamiltonian of the system is constructed, which takes into account the magneto-dipole nature of ions, electrons, atoms interaction and chemical catalytic bond between the reagents adsorbed on the surface of magnetoactive metal. To describe statistically and obtain reaction-diffusion equations, the main parameters of the abbreviated description are determined in the method of nonequilibrium statistical Zubarev operator [35,36]. The basic non-Markov transfer equations are obtained for the abbreviated description parameters of reaction-diffusion processes for magnetoactive ions and atoms adsorbed on the metal surface. Weakly nonequilibrium reactiondiffusion processes are also considered. In this case, the obtained system of transfer equations is closed and contains the non-dissipative and dissipative contributions, which describe non-Markov diffusion, magneto-diffusion and reaction catalytic processes. In the next article we will apply specifically the obtained transfer equations to description of hydrogen oxidation processes.

[1] Yartys V. A., Lototskyy M. V., Akiba E. et al. Magnesium based materials for hydrogen based energy storage: Past, present and future. International Journal of Hydrogen Energy. 44 (15), 7809-7859 (2019).

[2] Suchorski Yu., Datler M., Bespalov I., Zeininger J., Stöger-Pollach M., Bernardi J., Gronbech H., Rupprechter G. Surface-Structure Libraries: Multifrequential Oscillations in Catalytic Hydrogen Oxidation on Rhodium. J. Phys. Chem. C. 123 (7), 4217-4227 (2019).

[3] Cao L., Liu W., Luo Q. et al. Atomically dispersed iron hydroxide anchored on Pt for preferential oxidation of $\mathrm{CO}$ in $\mathrm{H}_{2}$. Nature. 565, 631-635 (2019).

[4] Gao C., Low J., Long R. et al. Heterogeneous Single-Atom Photocatalysts: Fundamentals and Applications. Chem. Rev. 120 (21), 12175-12216 (2020).

[5] Qiao B., Wang A., Yang X. et al. Single-atom catalysis of $\mathrm{CO}$ oxidation using $\mathrm{Pt}_{1} / \mathrm{FeO}_{x}$. Nature Chemistry. 3 (8), 634-641 (2011).

[6] Yakovkin I. N., Chernyi V. I., Naumovetz A. G. Effect of Li on the adsorption of CO and O on Pt. Journal of Physics D: Applied Physics. 32 (7), 841-844 (1999).

[7] Allian A. D., Takanabe K., Fujdala K. L. et al. Chemisorption of CO and Mechanism of CO Oxidation on Supported Platinum Nanoclusters. J. Am. Chem. Soc. 133 (12), 4498-4517 (2011).

[8] Michaelides A., Hu P. Catalytic water formation on platinum: A first-principles study. J. Am. Chem. Soc. 123 (18), 4235-4242 (2001).

[9] Völkening S., Bedörftig K., Jacobi K., Wintterlin J., Ertl G. Dual-path mechanism for catalytic oxidation of hydrogen on platinum surfaces. Phys. Rev. Lett. 83 (13), 2672-2675 (1999).

[10] Sachs C., Hildebrand M., Völkening S., Wintterlin J., Ertl G. Spatiotemporal self-organization in a surface reaction: From the atomic to the mesoscopic scale. Science. 293 (5535), 1635-1638 (2001).

[11] Sachs C., Hildebrand M., Völkening S., Wintterlin J., Ertl G. Reaction fronts in the oxidation of hydrogen on $\mathrm{Pt}(111)$ : Scanning tunneling microscopy experiments and reaction-diffusion modeling. J. Chem. Phys. 116, 5759-5773 (2002).

[12] Mitsui T., Rose M. K., Fomin E., Ogletree D. F., Salmeron M. A scanning tunneling microscopy study of the reaction between hydrogen and oxygen to form water on $\operatorname{Pd}(111)$. J. Chem. Phys. 117, 5855-5858 (2002).

[13] Wilke S., Natoli V., Cohen M. H. Theoretical investigation of water formation on Rh and Pt Surfaces. J. Chem. Phys. 112, 9986-9995 (2000).

[14] Schaak A., Shaikhutdinov S., Imbihl R. H/D-isotope effects in chemical wave propagation on surfaces: The $\mathrm{O}_{2}+\mathrm{H}_{2}$ and $\mathrm{NO}+\mathrm{H}_{2}$ reactions on $\mathrm{Rh}(110)$ and $\mathrm{Rh}(111)$. Surface Science. 421 (1-2), 191-203 (1999).

Mathematical Modeling and Computing, Vol. 8, No. 2, pp. 267-281 (2021) 
[15] Monine M. I., Schaak A., Rubinstein B. Y., Imbihl R., Pismen L. M. Dynamics of subsurface oxygen formation in catalytic water formation on a $\operatorname{Rh}\left(\begin{array}{llll}1 & 1 & 1\end{array}\right)$ surface - Experiment and simulation. Catalysis Today. 70 (4), 321-330 (2001).

[16] Suchorski Y., Rupprechter G. Heterogeneous surfaces as structure and particle size libraries of model catalysts. Catalysis Letters. 148, 2947-2956 (2018).

[17] Africh C., Lin H., Corso M., Esch F., Rosei R., Hofer W. A., Comelli G. Water Production Reaction on Rh(110). J. Am. Chem. Soc. 127 (32), 11454-11459 (2005).

[18] March N. H. Chemical Bonds Outside Metal Surfaces. Plenum Press, New York and London (1986).

[19] Smith J. R. (Ed.) Theory of Chemisorption. Springer-Verlag, Berlin, Heidelberg, New York (1980).

[20] Suhl H., Smith J. H., Kumar P. Role of Spin Fluctuations in the Desorption of Hydrogen from Paramagnetic Metals. Phys. Rev. Lett. 25 (20), 1442-1445 (1970).

[21] Yucel S. Theory of ortho-para conversion in hydrogen adsorbed on metal and paramagnetic surfaces at low temperatures. Phys. Rev. B. 39 (5), 3104-3115 (1989).

[22] Kato H. S., Okuyama H., Yoshnobu J., Kawai M. Estimation of direct and indirect interactions between CO molecules on Pd(1 110$)$. Surface Science. 513 (2), 239-248 (2002).

[23] Ziff R. M., Gulari E., Barshad Y. Kinetic phase transitions in an irreversible surface reaction model. Phys. Rev. Lett. 56 (24), 2553-2556 (1986).

[24] Zhdanov V. P. Surface restructuring kinetic oscillations and chaos in heterogeneous catalytic reactions. Phys. Rev. E. 59 (6), 6292-6305 (1999).

[25] Cisternas J., Keverkidis I., Li X. CO oxidation on thin Pt crystals: Temperature slaving and derivation of lumped models. J. Chem. Phys. 118 (7), 3312-3328 (2003).

[26] Kostrobii P. P., Tokarchuk M. V., Alekseyev V. I. Time evolution modelling of the surface cover for catalytic synthesis of ammonia. Preprint of the Institute for Condensed Matter Physics of the National Academy of Sciences of Ukraine, ICMP-03-28U (2003), (in Ukrainian).

[27] Nekhamkina O., Digilov R., Sheintuch M. Modeling of temporally complex breathing patterns during Pdcatalyzed CO oxidation. J. Chem. Phys. 119 (4), 2322-2332 (2003).

[28] Pavlenko N., Kostrobij P. P., Suchorski Yu., Imbihl R. Alkali metal effect on catalytic CO oxidation on a transition metal surface: a lattice-gas model. Surface Science. 489 (1-3), 29-36 (2001).

[29] Maximoff S. N., Head-Gordon M. P. Chemistry of fast electrons. Proceedings of the National Academy of Sciences. 106 (28), 11460-11465 (2009).

[30] Hervier A., Renzas J. R., Park J. Y., Somorjai G. A. Hydrogen oxidation-driven hot electron flow detected by catalytic nanodiodes. Nano letters. 9 (11), 3930-3933 (2009).

[31] Hervier A. Charge Transfer and Support Effects in Heterogeneous Catalysis. A dissertation submitted in partial satisfaction of the requirements for the degree of Doctor of Philosophy in Chemistry in the Graduate Division of the University of California, Berkeley (2011).

[32] Maximoff S. N., Head-Gordon M. Origin of fast electrons in catalytic hydrogen oxidation over platinum. arXiv: Materials Science (2014).

[33] Maximoff S. N. Role of charge transfer in catalytic hydrogen oxidation over platinum. arXiv preprint arXiv:1407.2570 (2016).

[34] Park J. Y., Baker L. R., Somorjai G. A. Role of hot electrons and metal-oxide interfaces in surface chemistry and catalytic reactions. Chemical reviews. 115 (8), 2781-2817 (2015).

[35] Zubarev D. N., Morozov V., Röpke G. Statistical mechanics of nonequilibrium Processes. Vol. 1, Basic Concepts, Kinetic Theory. Akad. Verl., Berlin (1996).

[36] Kostrobii P. P., Rudavskii Yu. K., Ignatyuk V. V., Tokarchuk M. V. Chemical reactions on adsorbinq surface kinetic level of description. Conden. Matter Phys. 6 (3), 409-423 (2003).

[37] Rudavskii Yu., Kostrobii P., Tokarchuk M., Batsevych O. Statistical theory of diffusion processes for magnetoactive particles adsorbed on the metal surface. Visnyk Natsionalnoho universytetu "Lvivska politekhnika". 540, 77-84 (2005), (in Ukrainian).

[38] Rudavskii Yu. K., Kostrobii P. P., Tokarchuk M. V., Batsevych O. F. Equations of reaction-diffusion processes for magnetoactive particles near the metal surface. Preprint of the Institute for Condensed Matter Physics of the National Academy of Sciences of Ukraine, ICMP-06-25U (2006), (in Ukrainian). 
[39] Mryglod I. M., Tokarchuk M. V. Hydrodynamic Theory of a Magnetic Liquid. Condens. Matter Phys. 3, 116-133 (1994).

[40] Mryglod I. M., Tokarchuk M. V., Folk R. On the hydrodynamic theory of a magnetic liquid. I. General description. Physica A. 220 (3-4), 325-348 (1995).

[41] Mryglod I. M., Tokarchuk M. V. Statistical hydrodynamics of magnetic fluids. I. The nonequilibrium statistical operator method. Theoretical and Mathematical Physics. 115, 479-495 (1998).

[42] Tokarchuk M. V., Kostrobii P. P., Humenyuk Y. A. Generalized transport equations of diffusion-reaction processes. The nonequilibrium statistical operator method. Journal of Physical Studies. 5 (2), 111-120 (2001).

[43] Kostrobii P. P., Tokarchuk M. V., Humenyuk Y. A. Nonequilibrium statistical operator method: Generalized transport equations of diffusion-reaction processes. Europ. Phys. Journal B - Condensed Matter and Complex Systems. 36 (4), 555-565 (2003).

[44] Kostrobij P. P., Tokarchuk M. V., Markovych B. M., Ihnatiuk V. V., Hnativ B. V. Reaktsiino-dyfuziini protsesy v systemakh "metal-gaz". Lviv, Lviv Polytechnic National University (2009), (in Ukrainian).

\title{
Статистичний підхід до теоретичного опису процесів каталітичної оксидації водню. Основні рівняння
}

\author{
Костробій П. П. ${ }^{1}$, Маркович Б. М. ${ }^{1}$, Рижа I. А. ${ }^{1}$, Токарчук М. В. ${ }^{2,1}$ \\ ${ }^{1}$ Націоналъний університет "Лъвівсъка політехніка", \\ вул. С. Бандери, 12, 79013, Львів, Україна \\ ${ }^{2}$ Інститут фізики конденсованих систем НАН Украӥни, \\ вул. Свенцічького, 1, 79011, Львів, Україна
}

\begin{abstract}
Запропоновано статистичний опис процесів каталітичної оксидації водню з врахуванням реакційно-дифузійних процесів для магнітоактивних іонів, атомів адсорбованих на поверхні металу. Отримано основні немарковські рівняння переносу для параметри скороченого опису реакційно-дифузійнійних процесів для магнітоактивних іонів, атомів адсорбованих на поверхні металу у методі нерівноважного статистичного оператора Зубарєва. Розглянуто також слабонерівноважні реакційно-дифузійні процеси.
\end{abstract}

Ключові слова: реакційно-дифузійні прочеси, немарковські рівняння, метод нерівноважного статистичного оператора. 